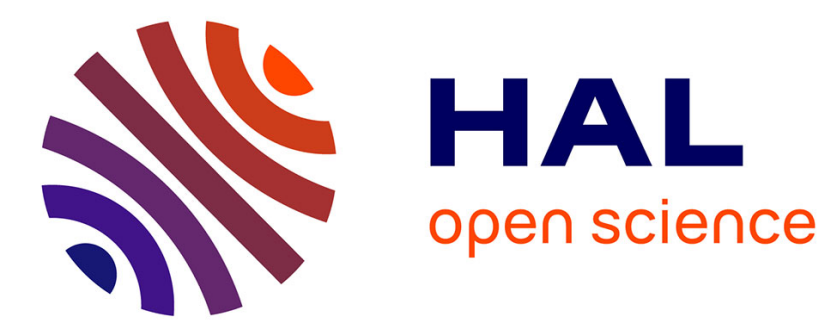

\title{
Prediction of hydration free energies for aliphatic and aromatic chloro derivatives using molecular dynamics simulations with the OPLS-AA force field
}

Oliver Beckstein, Bogdan Iorga

\section{- To cite this version:}

Oliver Beckstein, Bogdan Iorga. Prediction of hydration free energies for aliphatic and aromatic chloro derivatives using molecular dynamics simulations with the OPLS-AA force field. Journal of ComputerAided Molecular Design, 2012, 26 (5), pp.635-645. 10.1007/s10822-011-9527-9 . hal-02377115

\author{
HAL Id: hal-02377115 \\ https://hal.science/hal-02377115
}

Submitted on 22 Nov 2019

HAL is a multi-disciplinary open access archive for the deposit and dissemination of scientific research documents, whether they are published or not. The documents may come from teaching and research institutions in France or abroad, or from public or private research centers.
L'archive ouverte pluridisciplinaire HAL, est destinée au dépôt et à la diffusion de documents scientifiques de niveau recherche, publiés ou non, émanant des établissements d'enseignement et de recherche français ou étrangers, des laboratoires publics ou privés. 


\title{
Prediction of hydration free energies for aliphatic and aromatic chloro derivatives using molecular dynamics simulations with the OPLS-AA force field
}

\author{
Oliver Beckstein · Bogdan I. Iorga
}

Received: date / Accepted: date

\begin{abstract}
All-atom molecular dynamics computer simulations were used to blindly predict the hydration free energies of a range of chloro-organic compounds as part of the SAMPL3 challenge. All compounds were parameterized within the framework of the OPLS-AA force field, using an established protocol to compute the absolute hydration free energy via a windowed free energy perturbation approach and thermodynamic integration. Three different approaches to deriving partial charge parameters were pursued: (1) using existing OPLS-AA atom types and charges with minor adjustments of partial charges on equivalent connecting atoms; (2) calculation of quantum mechanical charges via geometry optimization, followed by electrostatic potential (ESP) fitting, using Jaguar at the LMP2/cc-pVTZ(-F) level; and (3) via geometry optimization and CHelpG charges (Gaussian03 at the HF/6-31G* level), followed by two-stage RESP fitting. Protocol 3 generated the most accurate predictions with a root mean square (RMS) error of $1.2 \mathrm{kcal} \cdot \mathrm{mol}^{-1}$ for the entire data set. It was found that the deficiency of the standard OPLS-AA parameters, protocol 1 (RMS error $2.4 \mathrm{kcal} \cdot \mathrm{mol}^{-1}$ overall), was mostly due to compounds with more than three chlorine substituents on an aromatic ring. For this latter subset, the RMS errors were $1.4 \mathrm{kcal} \cdot \mathrm{mol}^{-1}$ (protocol 3) and $4.3 \mathrm{kcal} \cdot \mathrm{mol}^{-1}$ (protocol 1), respectively. We propose new OPLS-AA atom types for aromatic carbon and chlorine atoms in rings with $\geq 4$ Cl-substituents that perform better than the best QM-based approach, resulting in an $\mathrm{RMS}$ error of $1.2 \mathrm{kcal} \cdot \mathrm{mol}^{-1}$ for these difficult compounds.

O. Beckstein

Structural Bioinformatics and Computational Biochemistry Unit, Department of Biochemistry, University of Oxford, Oxford OX1 3QU, UK

Present address: Department of Physics, Arizona State University, P.O. Box 871504, Tempe, AZ 852871504, USA

B.I. Iorga

Institut de Chimie des Substances Naturelles, CNRS UPR 2301, Centre de Recherche de Gif-sur-Yvette, 1 Avenue de la Terrasse, 91198 Gif-sur-Yvette, France

Tel.: +33169823094

Fax: +3316907 7247

E-mail: bogdan.iorga@icsn.cnrs-gif.fr
\end{abstract}


Keywords molecular dynamics - hydration free energy - OPLS-AA force field · ligand parameterization $\cdot$ free energy perturbation $\cdot$ thermodynamic integration

\section{Introduction}

The hydration free energy $\Delta G_{\text {hyd }}$, i.e. the change in Gibbs free energy for the transfer of a molecule from the gas phase to aqueous solution at constant temperature and pressure, is of fundamental importance to characterize the distribution of compounds in chemical and biological systems. It has also become a standard test case for the evaluation of the predictive power of quantitative computational methods. The SAMPL challenges (SAMPL1 [1], SAMPL2 [2], SAMPL3 in this issue) in particular asked participants to blindly predict hydration free energies for compounds whose unpublished experimental values were only revealed after submission of the predictions. This has turned out to be a fruitful approach to assess quantitative computational methods without bias and point out areas where improvements are required [3]. Solvation free energies have also been used to either parameterize computational models such as classical additive force fields used in molecular mechanics [4-6] or to evaluate the quality of existing force fields $[7,8]$.

Here we are focusing on classical all-atom molecular dynamics (MD) simulations in explicit solvent with additive and transferrable force fields. Our main interest is to assess the quality of parameterization of small molecules as such a parameterization is a crucial first step in studying the interactions of drug- or toxin-like molecules with biological systems. Methods based on classical all-atom MD simulations can provide precise predictions for hydration free energies [9] and current force fields are believed to be accurate to $1-2.5 \mathrm{kcal} \cdot \mathrm{mol}^{-1}$ for the prediction of hydration free energies of typical small organic molecules [3,10-16]

The data set provided for the SAMPL3 challenge consists of 36 chloro-organic compounds in three distinct chemical subsets with varying numbers of chlorine substituents. Understanding the relationship between structure and physico-chemical properties for this family of compounds is important in order to model the spread of these persistent and often toxic organic pollutants through the environment [17]. Subset 1 contains polychlorinated derivatives of ethane with all possible substitution combinations and up to six chlorine atoms (compounds 1-10, Figure 1). Polychlorinated derivatives of biphenyl in subset 2 (11-24, Figure 2) and of dibenzo- $p$-dioxin (subset 3, 25-36, Figure 3) contain aromatic ring systems with different substitution patterns for the chlorine substituents.

Computational studies predicting the hydration free energy for polychlorinated compounds are relatively rare in the literature. Previous work [18] described the prediction of hydration free energies for chlorinated ethanes using molecular dynamics simulations, in explicit solvent and with the OPLS force field. As the original parameters gave very poor results for the prediction of absolute and relative hydration free energies, the non-bonded $r^{12}$ coefficients for carbon, hydrogen and chlorine atoms were improved to reproduce experimental free energies of hydration [18]. More recently, hydration free energies of a data set of 57 polychlorobiphenyls (PCBs) were recently computed to a root mean square error (RMSE) of about $0.9 \mathrm{kcal} \cdot \mathrm{mol}^{-1}$ with 


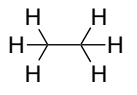

1

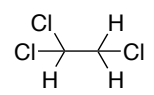

6

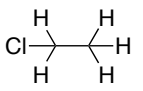

2

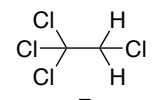

7

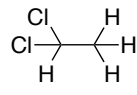

3

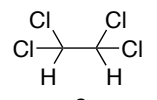

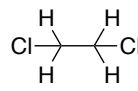

4
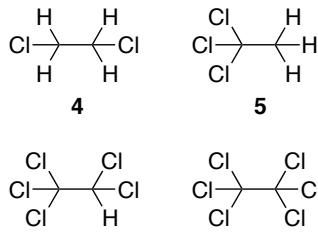

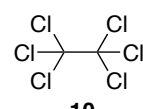

10

Fig. 1 Chemical structures of subset 1, containing chlorinated ethane derivatives.

an empirically corrected one-dimensional reaction site interaction model [19]. To our knowledge, no study dedicated to the prediction of hydration free energies of the polychlorinated dibenzo- $p$-dioxins (PCDD) family has been published to date.

Here we used all atom classical molecular dynamics (MD) simulations to compute the hydration energy from the interactions between the solute and water. These interactions are parameterized in a force field; for this work we used the OPLS-AA force field [5, 20-33] as a basis. The strongest non-bonded interaction in molecular systems is the electrostatic force, which is parameterized as a Coulomb force between point charges situated on the sites of the atoms. The point charges are typically not taken as full formal charges but as partial charges that, in the sum, reproduce the correct electrostatics of the molecule. In OPLS-AA, charges are determined for small model compounds and then directly transferred to an atom in another molecule that experiences the same chemical environment as the atom in the model compound. Water is a polar molecule and thus electrostatic interactions typically dominate the hydration free energy. Therefore, we evaluated three different protocols for generating the partial charges for the compounds 1-36. Protocol P1 essentially employed the native OPLS-AA charges whereas P2 and P3 computed the charges at the quantum mechanical (QM) level for each compound.

Comparison of the predicted hydration free energies with the hydration free energies released in the SAMPL3 evaluation phase (Figure 4) indicated that the QM partial charges produced better predictions than the OPLS-AA charges. In particular, compounds with at least 4 chlorine substituents on aromatic rings had much too negative hydration free energies in OPLS-AA. This observation prompted us to reparameterize the corresponding $\mathrm{Cl}$ and $\mathrm{C}$ partial charges, using the hydration free energy of the model compound hexachlorobenzene 37 (not in the test set) as a target. We show that the resulting parameterization protocol $\mathrm{P} 4$, which amounts to the introduction of two new atom types into the standard OPLS-AA parameterization protocol, performs better on the SAMPL3 set than any of our other approaches P1P3.

\section{Methods}

All compound structures were converted from SDF format to PDB format with CORINA version 3.44 (http://www.molecular-networks.com). Analysis of molecular dynamics trajectories was carried out with Gromacs tools (http://www.gromacs.org) 


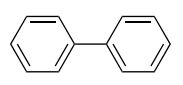

11<smiles>Clc1cc(Cl)c(-c2ccccc2)c(Cl)c1</smiles>

14<smiles>Clc1ccc(Cl)c(-c2cc(Cl)c(Cl)c(Cl)c2)c1</smiles>

17

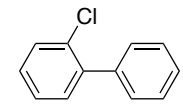

12
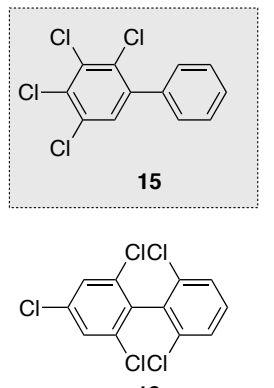

18

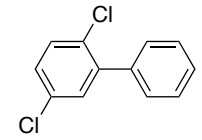

13<smiles>Clc1cccc(Cl)c1-c1c(Cl)cccc1Cl</smiles>

16

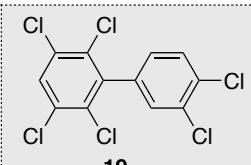

19

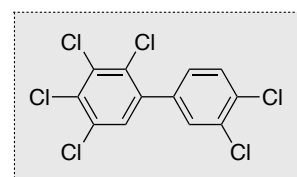

20

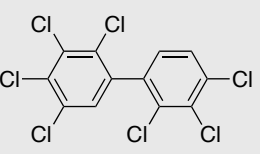

21

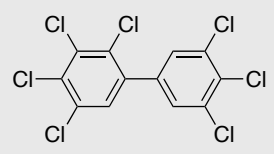

22

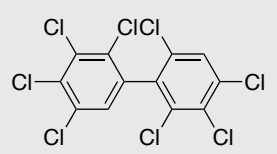

23

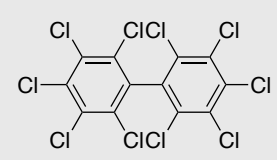

Fig. 2 Chemical structures of subset 2, containing polychlorinated biphenyl (PCB) derivatives. The highlighted compounds $\mathbf{1 5}$ and 19-24 contain aromatic ring systems with $\geq 4$ chlorine substituents. Hydrogen atoms are omitted for clarity.

[34] and MDAnalysis [35]. The OPLS-AA force field files that are part of Gromacs 4.5.3 [34] were used as a basis for the parameterization of the compounds 1-37.

\subsection{Parameterization protocols}

Topologies of all compounds were generated using an in-house developed script that assigns atom types based on the OPLS-AA force field. The hydration free energy depends strongly on the partial charges and hence three different protocols $\mathrm{P} 1-\mathrm{P} 3$ were tested for charge generation. A fourth protocol, P4, was used to derive optimised partial charge parameters for chlorine and carbon atoms that were insufficiently represented in the current OPLS-AA force field. The Lennard-Jones and bonded interactions were kept the same for protocols P1 to P4.

P1: OPLS-AA charges For parameterization protocol P1, the native charges from OPLS-AA were used. When these were missing, they were adapted from the existing 
<smiles>c1ccc2c(c1)Oc1ccccc1O2</smiles>

25<smiles>Clc1cc2c(cc1Cl)Oc1ccccc1O2</smiles>

28<smiles>Clc1c(Cl)c(Cl)c2c(c1Cl)Oc1ccccc1O2</smiles>

31<smiles>Clc1cccc2c1Oc1ccccc1O2</smiles>

26<smiles>Clc1ccc2c(c1)Oc1ccccc1O2</smiles>

27<smiles>Clc1cc(Cl)c2c(c1Cl)Oc1ccccc1O2</smiles>

30<smiles>Clc1cc2c(cc1Cl)Oc1cc(Cl)c(Cl)cc1O2</smiles>

33<smiles>Clc1ccc2c(c1)Oc1c(Cl)c(Cl)c(Cl)c(Cl)c1O2</smiles><smiles>Clc1ccc2c(c1)Oc1ccc(Cl)cc1O2</smiles>

29<smiles>Clc1ccc2c(c1)Oc1cc(Cl)c(Cl)c(Cl)c1O2</smiles>

32<smiles>Clc1cc2c(cc1Cl)Oc1c(Cl)c(Cl)c(Cl)c(Cl)c1Oc1c(Cl)c(Cl)c(Cl)c(Cl)c1O2</smiles>

Fig. 3 Chemical structures of subset 3, containing polychlorinated dibenzo- $p$-dioxin (PCDD) derivatives. The highlighted compounds $\mathbf{3 1}$ and 34-36 contain aromatic ring systems with $\geq 4$ chlorine substituents. Hydrogen atoms are omitted for clarity.

ones (e.g. atom type $o p l s_{-} 152$ with modified charges of 0.297 and 0.600 were used for $\mathrm{C}$ atoms in $\mathrm{RCHCl}_{2}$ and $\mathrm{RCCl}_{3}$, respectively; see Section 3.2 for a post-hoc validation of this approach).

P2: LMP2/cc-pVTZ(-F) QM charges For protocol P2, the charges were obtained after geometry optimization and electrostatic potential (ESP) fitting, using Jaguar (http://www.schrodinger.com) at the LMP2/cc-pVTZ(-F) level. This methodology has been previously used for the OPLS-AA force field development (protein amino acids and small organic molecules) $[21,26,27]$. The charges for chemically equivalent atoms obtained from the Jaguar calculations output were generally not identical, and they were adjusted manually before further use.

P3: HF/6-31G* QM charges For P3, the charges were obtained after geometry optimization and $\mathrm{CHelpG}$ charges calculation using Gaussian03 (http://www.gaussian.com) [36] at the HF/6-31G* level, then a two-stage RESP fitting using AmberTools (http://ambermd.org). This methodology has been previously used for heterocycles parameterization in the OPLS-AA force field [23, 24].

P4: New OPLS-AA parameters based on the hydration free energy of hexachlorobenzene New OPLS-AA atom types for chlorine and carbon were developed for the case of aromatic rings with four or more chlorine substituents, using the model compound hexachlorobenzene 37 (Figure 5A). Lennard-Jones and bonded interactions were retained from OPLS-AA atom types opls_264 (Cl) and opls_263(C), which were 
originally derived from chlorobenzene [37]. The partial charges of the $\mathrm{Cl}$ and the $\mathrm{C}$ atom were set to equal but opposite values $\left(q_{\mathrm{Cl}}=-q_{\mathrm{C}}\right)$. They were adjusted until the computed hydration free energy matched the experimental estimate, $\Delta G_{\text {hyd }}^{\exp }=-2.26$ $\mathrm{kcal} / \mathrm{mol}$, which had been calculated as the mean from two different experimental measurements [19].

\subsection{Hydration free energy calculation}

Hydration free energies were calculated via free energy perturbation (FEP) molecular dynamics (MD) simulations of each molecule in a water box. All simulations were performed with Gromacs 4.5.3 [34]. Processing and analysis of the free energy simulations was carried out with scripts based on the GromacsWrapper Python tool kit (O. Beckstein, https://github.com/orbeckst/GromacsWrapper).

The FEP protocol follows the works of Mobley and colleagues [10], with some small differences. A molecule was solvated with TIP4P water [38] in a dodecahedral periodic simulation cell with at least $1.0 \mathrm{~nm}$ between the solute and the box surfaces. Initial equilibrium $N P T$ MD simulation $(T=300 \mathrm{~K}, P=1$ bar) of the molecule were run for $50 \mathrm{~ns}$. The simulations were run as Langevin dynamics (integration time step $2 \mathrm{fs}$ ) for temperature control, with the friction coefficient for each particle computed as mass/0.1 ps. The average pressure was held constant with an isotropic Berendsen barostat (relaxation time constant $\tau_{p}=1 \mathrm{ps}$ and compressibility $\kappa_{p}=4.6 \times 10^{-5} \mathrm{bar}^{-1}$ ). The grid-based neighbor list was updated every five time steps. Lennard-Jones interactions were calculated up to a cutoff of $1 \mathrm{~nm}$ and a dispersion correction (implemented in Gromacs) was applied to energy and pressure to account for van der Waals interactions beyond the cutoff in a mean field manner. Coulomb interactions were handled with the SPME method [39] (short range cutoff $1 \mathrm{~nm}, 0.12 \mathrm{~nm}$ Fourier grid spacing, sixth order spline interpolation). Bonds containing hydrogen atoms were constrained with the P-LINCS algorithm [40] (fourth order expansion with a single iteration).

The last frame of the NPT simulation was used as the input for the free energy perturbation (FEP) simulations. FEP calculations were performed in the $N V T$ ensemble without a barostat but used the same parameters as the NPT simulations with the exception of FEP specific alterations and a higher P-LINCS order of 12.

Simulations were run on up to eight cores. The domain decomposition algorithm in Gromacs 4.5.3 did not always handle well the simulations for these relatively small systems and therefore most of them were run with the particle-based decomposition. The simulation system sizes were fairly small (8-22 atoms for solute and 1100-2600 atoms for solvent) and did not substantially benefit from parallelizing a single simulation further. However, the automated FEP protocol described below requires 21 simulations that can all be carried out independently. One eight-core workstation was sufficient to calculate one hydration free energy in 1-2 days, depending on the size of the system.

The FEP protocol computes the free energy difference between the solvated molecule and the molecule in the gas phase by constructing an alchemical (non-physical) pathway during which the interactions of the molecule with the solvent are stepwise de- 
coupled. A coupling parameter $\lambda$ for each interaction is used to scale the interaction: $\lambda=0$ indicates that the interaction is at full strength and at 1 it is switched off. Coulomb interactions were linearly reduced to zero over five windows (coupling parameter $\lambda_{\text {Coul }} \in\{0,0.25,0.5,0.75,1\}$ ) while the van der Waals (Lennard-Jones) interactions were maintained (i.e. $\lambda_{\mathrm{vdW}}=0$ ); sixteen windows were used to switch off the Lennard-Jones term for the uncharged solute $\left(\lambda_{\text {Coul }}=1\right.$ and $\lambda_{\mathrm{vdW}} \in\{0,0.05$, $0.1,0.2,0.3,0.4,0.5,0.6,0.65,0.7,0.75,0.8,0.85,0.9,0.95,1\})$. Each window was simulated for $5 \mathrm{~ns}$. The van der Waals calculations used soft core potentials with the values suggested by [10] ( $\alpha=0.5$, power 1 , and $\sigma=0.3 \mathrm{~nm})$. The calculations made use of the "couple-intramol = no" feature in Gromacs [34] (new in release 4.x), which performs a decoupling simulation that leaves intra-molecular interactions unchanged and thus avoids having to calculate gas-phase contributions separately. The derivative of the Hamiltonian $\mathscr{H}$ with respect to the coupling parameter $\lambda, \partial \mathscr{H} / \partial \lambda$, was saved for every time step. Thermodynamic integration (TI) over the average derivatives of all windows,

$$
\Delta G=\int_{0}^{1}\left\langle\frac{\partial \mathscr{H}}{\partial \lambda}\right\rangle d \lambda
$$

yielded the free energy contributions for the decharging and decoupling steps. Eq. 1 was integrated numerically with the composite Simpson's rule [41] as implemented in SciPy (http://www.scipy.org).

The total hydration free energy (transfer from gas phase to aqueous phase at the $1 \mathrm{M} / 1 \mathrm{M}$ Ben-Naim standard state) is calculated as $\Delta G_{\mathrm{hyd}}=-\left(\Delta G_{\mathrm{Coul}}+\Delta G_{\mathrm{vdW}}\right)$. Note that any corrections for simulating at constant volume have been neglected (and hence technically our transfer energy is a Helmholtz free energy). In the last stages of preparation of this manuscript for submission, these corrections have been evaluated more in depth and we realized that they can be significant in some cases. Preliminary results indicate that correct treatment of these corrections will substantially improve our results. Additional calculations are currently running to address this issue.

The error on the hydration free energy is estimated from the errors of the individual $\langle\partial \mathscr{H} / \partial \lambda\rangle$ of each FEP window. The error $\delta$ of the mean $\langle\partial \mathscr{H} / \partial \lambda\rangle$ is calculated as

$$
\delta=\sqrt{2 t_{c} C(0) \tau^{-1}}
$$

where $C(t)$ is the autocorrelation function of the fluctuations around the mean, $\partial \mathscr{H} / \partial \lambda-$ $\langle\partial \mathscr{H} / \partial \lambda\rangle, t_{c}$ the correlation time (assuming an inital exponential decay of the autocorrelation function $\left.C(t) \sim \exp \left(-t / t_{c}\right)\right)$, and $\tau$ the total length of the simulation [42]. The error on each TI integral is calculated analytically via propagation of errors through Simpson's rule (implemented in GromacsWrapper as an extension of the SciPy functionality for Simpson's rule). The final error on $\Delta G_{\mathrm{hyd}}$ is $\left(\delta_{\mathrm{Coul}}^{2}+\delta_{\mathrm{vdW}}^{2}\right)^{1 / 2}$.

\section{Results and Discussion}

The main results of the hydration free energy calculations are summarized in Figure 4 and detailed in Table 1. 
Table 1 Computed and experimental hydration free energies $\Delta G_{\text {hyd }}$ with error estimate (in $\mathrm{kcal} \cdot \mathrm{mol}^{-1}$ ) for all compounds (denoted by "id"). The absolute difference between computed and experimental hydration free energy is shown for each compound and parameterization protocol $i$ as $D_{\text {id }}^{(i)}=\left|\Delta G_{\text {hyd,id }}^{(i)}-\Delta G_{\text {hyd,id }}^{\exp }\right|$. The standard error in the last significant digits is given in parentheses. The root mean square error (RMSE) $\sqrt{N^{-1} \sum_{\mathrm{id}}^{N} D_{\mathrm{id}}^{2}}$ is listed for chemically distinct classes of compounds (see text).

\begin{tabular}{|c|c|c|c|c|c|c|c|c|c|}
\hline \multirow[t]{2}{*}{ id } & \multirow{2}{*}{$\begin{array}{c}\text { Exp. } \\
\Delta G_{\text {hyd }}^{\text {exp }}\end{array}$} & \multicolumn{2}{|c|}{ Protocol 1} & \multicolumn{2}{|c|}{ Protocol 2} & \multicolumn{2}{|c|}{ Protocol 3} & \multicolumn{2}{|c|}{ Protocol 4} \\
\hline & & $\Delta G_{\text {hyd }}^{(1)}$ & $D^{(1)}$ & $\Delta G_{\text {hyd }}^{(2)}$ & $D^{(2)}$ & $\Delta G_{\text {hyd }}^{(3)}$ & $D^{(3)}$ & $\Delta G_{\text {hyd }}^{(4)}$ & $D^{(4)}$ \\
\hline 1 & $1.87(10)$ & $2.69(3)$ & 0.82 & $2.27(3)$ & 0.40 & $2.49(3)$ & 0.62 & & \\
\hline 2 & $-0.39(10)$ & $0.37(4)$ & 0.76 & $0.17(4)$ & 0.56 & $0.19(4)$ & 0.58 & & \\
\hline 3 & $-0.88(10)$ & $-0.14(5)$ & 0.74 & $-0.08(4)$ & 0.80 & $0.42(4)$ & 1.30 & & \\
\hline 4 & $-1.80(10)$ & $-0.42(7)$ & 1.38 & $0.09(6)$ & 1.89 & $-0.73(6)$ & 1.07 & & \\
\hline 5 & $-0.26(10)$ & $0.10(5)$ & 0.36 & $0.84(5)$ & 1.10 & $0.11(5)$ & 0.37 & & \\
\hline 6 & $-1.97(10)$ & $-1.64(20)$ & 0.33 & $-0.54(6)$ & 1.43 & $-0.08(7)$ & 1.89 & & \\
\hline 7 & $-1.43(10)$ & $-0.99(5)$ & 0.44 & $-0.23(5)$ & 1.20 & $-0.17(5)$ & 1.26 & & \\
\hline 8 & $-2.37(10)$ & $-2.53(14)$ & 0.16 & $0.16(8)$ & 2.53 & $-0.23(9)$ & 2.14 & & \\
\hline 9 & $-1.23(10)$ & $-2.35(6)$ & 1.12 & $0.63(6)$ & 1.86 & $-0.70(6)$ & 0.53 & & \\
\hline 10 & $-0.64(10)$ & $0.01(7)$ & 0.65 & $-0.37(7)$ & 0.27 & $0.83(6)$ & 1.47 & & \\
\hline \multirow{3}{*}{\multicolumn{2}{|c|}{$\begin{array}{l}\text { RMSE 1-10 } \\
\text { RMSE 1,2,4 } \\
\text { RMSE 3,5-10 }\end{array}$}} & & 0.76 & & 1.39 & & 1.26 & & \\
\hline & & & 1.03 & & & & & & \\
\hline & & & 0.62 & & & & & & \\
\hline 11 & $-2.23(10)$ & $-2.42(7)$ & 0.19 & $-4.28(8)$ & 2.05 & $-1.60(7)$ & 0.63 & & \\
\hline 12 & $-2.69(10)$ & $-0.58(8)$ & 2.11 & $-3.24(7)$ & 0.55 & $-0.76(7)$ & 1.93 & & \\
\hline 13 & $-2.46(10)$ & $-2.83(8)$ & 0.37 & $-3.55(8)$ & 1.09 & $-1.35(8)$ & 1.11 & & \\
\hline 14 & $-2.16(10)$ & $-2.56(8)$ & 0.40 & $-1.99(8)$ & 0.17 & $-0.78(9)$ & 1.38 & & \\
\hline 15 & $-3.48(1.00)$ & $-4.48(9)$ & 1.00 & $-3.00(9)$ & 0.48 & $-2.80(9)$ & 0.68 & $-2.40(9)$ & 1.08 \\
\hline 16 & $-2.28(12)$ & $-1.71(10)$ & 0.57 & $-2.17(9)$ & 0.11 & $-1.82(10)$ & 0.46 & & \\
\hline 17 & $-3.61(13)$ & $-3.77(9)$ & 0.16 & $-2.08(9)$ & 1.53 & $-2.74(9)$ & 0.87 & & \\
\hline 18 & $-1.96(10)$ & $-2.89(10)$ & 0.93 & $0.23(9)$ & 2.19 & $-1.61(10)$ & 0.35 & & \\
\hline 19 & $-4.38(22)$ & $-4.97(11)$ & 0.59 & $-1.62(10)$ & 2.76 & $-2.20(10)$ & 2.18 & $-2.97(11)$ & 1.41 \\
\hline 20 & $-3.04(10)$ & $-6.47(11)$ & 3.43 & $-1.35(9)$ & 1.69 & $-2.76(10)$ & 0.28 & $-3.01(10)$ & 0.03 \\
\hline 21 & $-4.40(10)$ & $-7.93(10)$ & 3.53 & $0.27(10)$ & 4.67 & $-3.38(11)$ & 1.02 & $-4.48(11)$ & 0.08 \\
\hline 22 & $-3.17(10)$ & $-8.04(11)$ & 4.87 & $-0.97(11)$ & 2.20 & $-1.65(11)$ & 1.52 & $-4.97(10)$ & 1.80 \\
\hline 23 & $-4.61(25)$ & $-7.36(11)$ & 2.75 & $-0.49(13)$ & 4.12 & $-2.43(10)$ & 2.18 & $-2.59(11)$ & 2.02 \\
\hline 24 & $-2.98(1.00)$ & $-10.38(12)$ & 7.40 & $-0.55(11)$ & 2.43 & $-2.53(12)$ & 0.45 & $-3.59(11)$ & 0.61 \\
\hline \multicolumn{3}{|c|}{ RMSE 11-24 } & 2.90 & & 2.29 & & 1.25 & & \\
\hline \multicolumn{2}{|c|}{ RMSE 15,19-24 } & & 4.31 & & 3.17 & & 1.51 & & 1.35 \\
\hline 25 & $-3.15(10)$ & $-2.13(7)$ & 1.02 & $-3.83(7)$ & 0.68 & $-2.19(7)$ & 0.96 & & \\
\hline 26 & $-3.52(10)$ & $-0.97(7)$ & 2.55 & $-3.03(8)$ & 0.49 & $-3.22(8)$ & 0.30 & & \\
\hline 27 & $-3.10(10)$ & $-2.10(8)$ & 1.00 & $-3.64(8)$ & 0.54 & $-2.02(7)$ & 1.08 & & \\
\hline 28 & $-3.56(1.00)$ & $-3.08(9)$ & 0.48 & $-2.59(8)$ & 0.97 & $-2.45(8)$ & 1.11 & & \\
\hline 29 & $-3.67(12)$ & $-1.91(8)$ & 1.76 & $-2.72(9)$ & 0.95 & $-2.42(9)$ & 1.25 & & \\
\hline 30 & $-4.05(10)$ & $-3.88(9)$ & 0.17 & $-1.33(9)$ & 2.72 & $-2.27(9)$ & 1.78 & & \\
\hline 31 & $-3.81(14)$ & $-6.77(10)$ & 2.96 & $-2.40(10)$ & 1.41 & $-2.31(9)$ & 1.50 & $-3.10(9)$ & 0.71 \\
\hline 32 & $-3.84(1.00)$ & $-3.43(9)$ & 0.41 & $-3.74(9)$ & 1.00 & $-3.03(9)$ & 0.81 & & \\
\hline 33 & $-3.37(1.00)$ & $-3.83(9)$ & 0.46 & $-2.53(10)$ & 0.84 & $-3.16(9)$ & 0.21 & & \\
\hline 34 & $-4.15(1.00)$ & $-6.12(10)$ & 1.97 & $-2.23(9)$ & 1.92 & $-3.31(10)$ & 0.84 & $-2.58(10)$ & 1.57 \\
\hline 35 & $-3.71(1.00)$ & $-7.62(10)$ & 3.91 & $-2.82(10)$ & 0.89 & $-4.87(10)$ & 1.16 & $-4.74(11)$ & 1.03 \\
\hline 36 & $-4.53(1.00)$ & $-11.23(12)$ & 6.70 & $-1.80(11)$ & 2.73 & $-3.37(11)$ & 1.16 & $-4.32(11)$ & 0.21 \\
\hline \multicolumn{3}{|c|}{$\begin{array}{l}\text { RMSE 25-36 } \\
\text { RMSE 31 34-36 }\end{array}$} & 2.66 & & 1.44 & & 1.10 & & \\
\hline $\mathrm{RM}$ & E 31,34-36 & & 4.27 & & 1.87 & & 1.19 & & 1.01 \\
\hline \multicolumn{3}{|c|}{ RMSE 1-36 } & 2.41 & & 1.80 & & 1.21 & & \\
\hline \multicolumn{3}{|c|}{ RMSE 15,19-24,31,34-36 } & 4.29 & & 2.72 & & 1.39 & & 1.22 \\
\hline
\end{tabular}



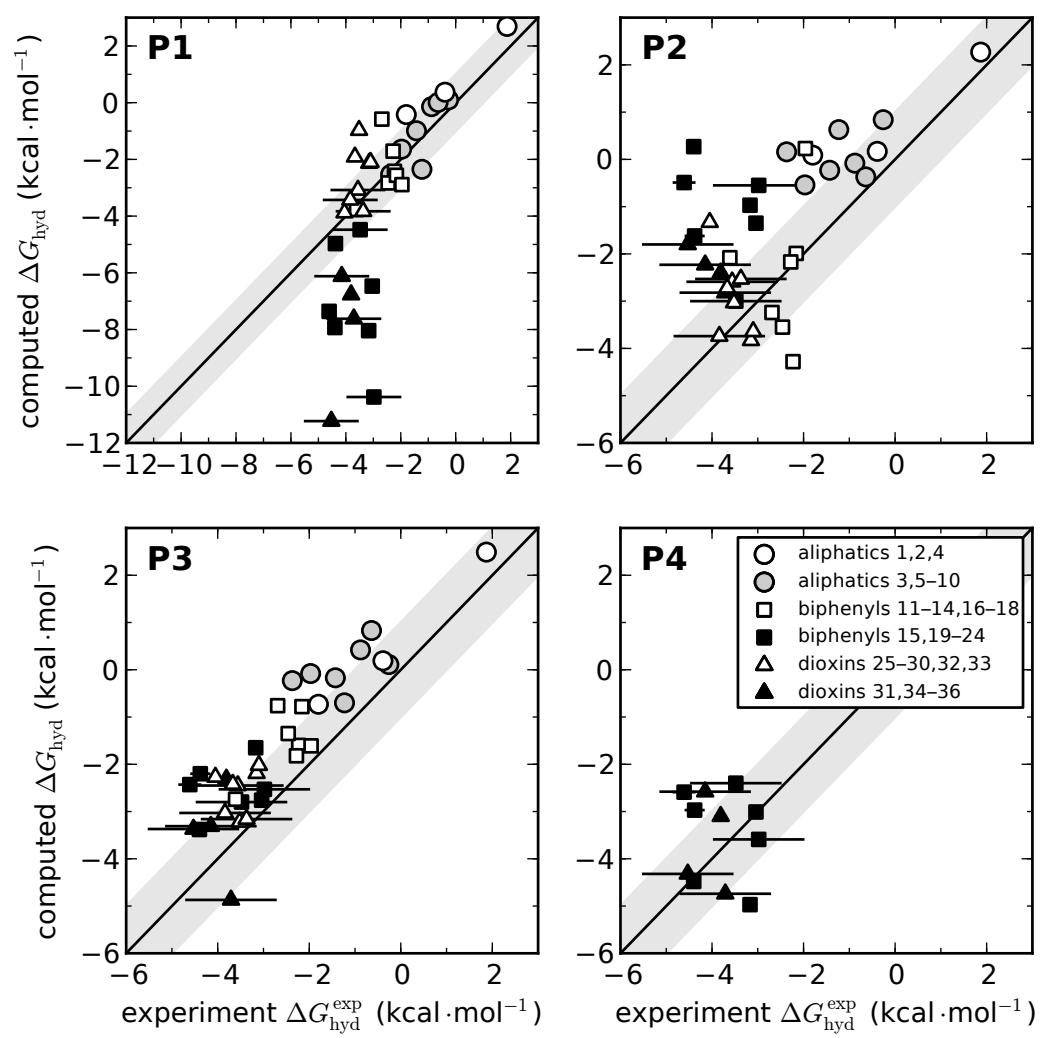

Fig. 4 Correlation between experimental and computed hydration free energies. The computed $\Delta G_{\text {hyd }}$ is shown against the experimental value for each parameterization protocol P1-P4. Perfect agreement is shown by the diagonal line in each plot and $\pm 1 \mathrm{kcal} \cdot \mathrm{mol}^{-1}$ from ideal is indicated by the shaded area. Error bars denote the experimental or computational error at one standard deviation from the mean. Computational errors are typically smaller than the marker symbols $\left(<0.15 \mathrm{kcal} \cdot \mathrm{mol}^{-1}\right)$. The plot range for P1 is expanded compared to the other protocols in order to accommodate the predictions for compounds with $\geq 4$ chlorine substituents on aromatic rings that deviate substantially from the experimental values (black filled markers).

\subsection{Predicted hydration free energies}

For each molecule, hydration free energy calculations were carried out using the three topologies generated with protocols P1-P3. The corresponding three sets of results $\left(\Delta G_{\text {hyd }}^{(1)}\right.$ to $\Delta G_{\text {hyd }}^{(3)}$ in Table 1$)$ were submitted to the SAMPL3 challenge. The accuracy of the computed $\Delta G_{\text {hyd }}$ was quantified by computing the root mean square error (RMSE) from the experimental hydration free energies, which were released by the SAMPL3 organisers after the submission of the predictions. A RMSE to within 
Table 2 New OPLS-AA parameters for carbon atoms in $\mathrm{RCHCl}_{2}$ and $\mathrm{RCCl}_{3}$ (oplsa_152A and oplsa_152B) and carbon (oplsa_263A) and chlorine (oplsa_264A) atoms in aromatic rings with $\geq 4$ chlorine substituents.

\begin{tabular}{llrrrrll}
\hline name $^{\mathrm{a}}$ & type $^{\mathrm{b}}$ & $Z^{\mathrm{c}}$ & $m(\mathrm{u})^{\mathrm{d}}$ & $q(e)^{\mathrm{e}}$ & $\sigma(\mathrm{nm})^{\mathrm{f}}$ & $\varepsilon\left(\mathrm{kJ} \cdot \mathrm{mol}^{-1}\right)^{\mathrm{g}}$ & \\
\hline opls_152A & CT & 6 & 12.011 & 0.297 & 0.350 & 0.276144 & $; \mathrm{C}$ in $\mathrm{RCHCl} 2$ \\
opls_152B & CT & 6 & 12.011 & 0.600 & 0.350 & 0.276144 & $; \mathrm{C}$ in $\mathrm{RCCl} 3$ \\
opls_263A & CA & 6 & 12.011 & 0.090 & 0.355 & 0.29288 & $; \mathrm{C}(\mathrm{Cl})$ perchlorobenzenes \\
opls_264A & Cl & 17 & 35.453 & -0.090 & 0.340 & 1.25520 & $;$ Cl perchlorobenzenes \\
\hline
\end{tabular}

${ }^{\mathrm{a}}$ proposed OPLS-AA atom type name $\quad{ }^{\mathrm{b}}$ bonded type $\quad{ }^{\mathrm{c}}$ atomic number

$\mathrm{d}$ atomic mass in atomic mass constants $m_{\mathrm{u}}=1.660538921 \times 10^{-27} \mathrm{~kg}$

${ }^{\mathrm{e}}$ partial charge in elementary charges $e=1.602176565 \times 10^{-19} \mathrm{C}$

${ }^{f}$ length parameter of the OPLS-AA Lennard-Jones potential $\left.V_{\mathrm{LJ}}(r)=4 \varepsilon\left[(\sigma / r)^{12}-(\sigma / r)^{6}\right)\right]$ [5]

$\mathrm{g}$ energy well depth of the OPLS-AA Lennard-Jones potential $V_{\mathrm{LJ}}(r)$

chemical accuracy, i.e. about $1 \mathrm{kcal} \cdot \mathrm{mol}^{-1}$, can be considered sufficient for many applications [3].

At the time of submission, experimental values for the hydration free energy were known for two compounds from this dataset: ethane $\mathbf{1}$ and hexachloroethane 10, the latter being present in the SAMPL2 data set [2]. For both compounds, the second protocol (P2) gave the best results (RMSE $0.3 \mathrm{kcal} \cdot \mathrm{mol}^{-1}$ ) and thus from this very limited data set, $\mathrm{P} 2$ appeared to be the most promising approach. However, comparison of the predictions for the whole data set 1-36 to the released experimental data (Figure 4) showed that P3 (QM charges from Gaussian03) produced the best blind predictions with a RMSE of $1.2 \mathrm{kcal} \cdot \mathrm{mol}^{-1}$ compared to $1.8 \mathrm{kcal} \cdot \mathrm{mol}^{-1}$ for P2 (QM charges from Jaguar). Compared to the QM-derived charges, the standard OPLS-AA approach (P1) was less accurate with RMSE $2.4 \mathrm{kcal} \cdot \mathrm{mol}^{-1}$ (Table 1).

A more detailed analysis in terms of distinct chemical groups showed a more diverse picture. In subset 1, which contains the chloro ethane derivatives 1-10, the trend was completely reversed. In this case, protocol P1 provided the most accurate predictions, with a RMSE value of $0.8 \mathrm{kcal} \cdot \mathrm{mol}^{-1}$. The QM-based methods did show smaller errors for the more complicated compounds in subsets 2 (11-24) and 3 (2536) than the standard OPLS-AA charges where P3 produced more accurate results than P2 or P1 (Table 1).

The encouraging results for the standard OPLS-AA force field for subset 1 suggested that the force field is able to reach near chemical accuracy for chloro-organic compounds and its failure to do so for subsets 2 and 3 might have a cause lying in the chemical structure of the compounds. It turned out that compounds with at least four chlorine substituents on an aromatic ring $(\mathbf{1 5}, \mathbf{1 9}-\mathbf{2 4}, \mathbf{3 1}, \mathbf{3 4}-\mathbf{3 6})$ had substantially too negative hydration free energies (RMSE $4.3 \mathrm{kcal} \cdot \mathrm{mol}^{-1}$ compared to the best RMSE $1.4 \mathrm{kcal} \cdot \mathrm{mol}^{-1}$ for P3). This was particularly obvious when the correlation between experimental and computed values was plotted as in Figure 4. These results prompted us to develop new force field parameters for these difficult compounds.

3.2 Parameterization and validation of new OPLS-AA atom types 
A<smiles>Clc1c(Cl)c(Cl)c(Cl)c(Cl)c1Cl</smiles>

37

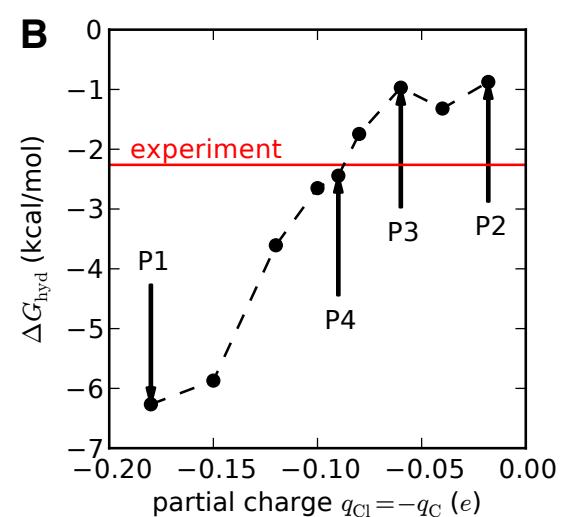

Fig. 5 Model compound hexachlorobenzene (37). A: chemical structure. B: Calculated hydration free energy $\Delta G_{\mathrm{hyd}}$ as a function of the partial charge $q_{\mathrm{Cl}}$ on the chlorine substituent or $-q_{\mathrm{Cl}}$ on the $\mathrm{C}_{\mathrm{ipso}}$ atom bearing the chlorine substituent. The red line shows the average of the known experimental values, $-2.26 \mathrm{kcal} / \mathrm{mol}$ [19]. Arrows indicate values generated from the protocols P1-P3 discussed in the text. The new value $q_{\mathrm{Cl}}$ proposed for OPLS-AA is indicated by $\mathrm{P} 4$.

Parameterization of aliphatic C atoms in $\mathrm{RCHCl}_{2}$ and $\mathrm{RCCl}_{3}$ In the original OPLSAA force field, no parameters were available for aliphatic carbon atoms bearing two or three chlorine substituents, only aliphatic $\mathrm{C}$ atoms of $\mathrm{RCH}_{2} \mathrm{Cl}$ type being parameterized (available as opls_152), starting from 1,2-dichloroethane [37]. As several compounds from the SAMPL3 dataset (3,5-10) contained the $\mathrm{RCHCl}_{2}$ and $\mathrm{RCCl}_{3}$ motifs, two new atom types were defined starting from opls_152 and following the same OPLS-AA philosophy. Consequently, modified charges of 0.297 and 0.600 were used in protocol $\mathrm{P} 1$ for $\mathrm{C}$ atoms in $\mathrm{RCHCl}_{2}$ and $\mathrm{RCCl}_{3}$, respectively, all other parameters being identical with opls_l52 (Table 2). The values of these charges were chosen in order to conserve the overall neutrality of the molecule and make use of the existing atom types [37] for hydrogen and chlorine atoms in alkyl chlorides (opls_153 and $o p l s_{-} 151$, respectively). The predictions for the compounds containing these new atom types $(\mathbf{3 , 5}-\mathbf{1 0})$ were in very good agreement with the experimental hydration free energy values (RMSE $0.62 \mathrm{kcal} \cdot \mathrm{mol}^{-1}$, see Table 1 ), better than the predictions for the compounds making use of "classical" OPLS-AA parameters (1-2,4, RMSE $\left.1.03 \mathrm{kcal} \cdot \mathrm{mol}^{-1}\right)$. In these conditions, we believe that these two new atom types are validated and appropriate for further use in molecular dynamics simulations with the OPLS-AA force field.

Parameterization of $\mathrm{Cl}$ atoms in aromatic rings with $\geq 4$ chloro substituents Three different atom types are available in the OPLS-AA force field for fluorobenzenes (opls_719, opls_728 and opls_721, defined for systems with one, two and six fluorine substituents on the aromatic ring, respectively). On the other hand, only one atom type (opls_264) is available for chlorobenzenes, corresponding to the monosubstituted aromatic rings [37]. As already stressed above, the compounds with four or more chloro substituents on the aromatic ring $(\mathbf{1 5}, \mathbf{1 9 - 2 4 , 3 1 , 3 4 - 3 6 )}$ contributed most to the overall prediction error for the hydration free energy in the SAMPL3 
dataset (Table 1 and Figure 4). A possible explanation for these results is that the opls_264 atom type is not appropriate for use with these highly substituted systems, possibly due to interactions between the chlorine atoms as also found in a different study [19]. Within the SAMPL3 challenge, we were able to obtain improved predictions for these difficult compounds using modified charges generated from QM calculations (protocols P2 and P3, with RMSE $2.72 \mathrm{kcal} \cdot \mathrm{mol}^{-1}$ and $1.39 \mathrm{kcal} \cdot \mathrm{mol}^{-1}$, respectively, compared with $4.29 \mathrm{kcal} \cdot \mathrm{mol}^{-1}$ using protocol P1). However, with protocols $\mathrm{P} 2$ and $\mathrm{P} 3$ the charge on $\mathrm{Cl}$ atoms always differs according to their environment within the molecules. Such variable charges are not compatible with the OPLS-AA philosophy and makes it impossible to transfer these charges to other, chemically similar compounds. With approaches P2 and P3, a full QM calculation would have to be carried out for each new compound of interest. The SAMPL3 data provided an opportunity to define a new transferrable OPLS-AA atom type for $\mathrm{Cl}$ atoms in highly substituted aromatic systems (perchlorobenzenes). We used hexachlorobenzene (37, Figure 5A), which is not included in the SAMPL3 data set, but was present in the SAMPL2 data set [2], as a model compound. Starting from the standard OPLS-AA atom type opls_264 for $\mathrm{Cl}$ (protocol P1), we computed $\Delta G_{\text {hyd }}$ as a function of the partial charge $q_{\mathrm{Cl}}$ on the $\mathrm{Cl}$ atom (Figure $5 \mathrm{~B}$ ). The aromatic $\mathrm{C}_{\mathrm{ipso}}$ atom bearing the $\mathrm{Cl}$ substituent was based on opls_263 and was assigned the same charge as the $\mathrm{Cl}$ atom but with opposite sign $\left(q_{\mathrm{C}_{\mathrm{ipso}}}=-q_{\mathrm{Cl}}\right)$. As a target value for $\Delta G_{\text {hyd }}$ we employed the experimental estimate of $-2.26 \mathrm{kcal} \cdot \mathrm{mol}^{-1}$, which was also used by Ratkova et al [19]. The standard OPLS-AA parameters $\left(q_{\mathrm{Cl}}=-0.180\right)$ yielded a $\Delta G_{\text {hyd }}$ too negative by $4.01 \mathrm{kcal} \cdot \mathrm{mol}^{-1}$, consistent with the general trend observed for the aromatic compounds (11-36) from the SAMPL3 set (P1). The QM approaches produced smaller $\mathrm{Cl}$ charges of -0.060 (P3) and -0.018 (P2), leading to $\Delta G_{\text {hyd }}$ being too positive by 1.29 and $1.39 \mathrm{kcal} \cdot \mathrm{mol}^{-1}$, respectively. We then systematically varied $q_{\mathrm{Cl}}$ to match the target hydration free energy and finally chose $q_{\mathrm{Cl}}=-0.090$ with a signed deviation of $-0.18 \mathrm{kcal} \cdot \mathrm{mol}^{-1}$ to define new atom types opls_263A $\left(\mathrm{C}_{\mathrm{ipso}}\right)$ and opls_264A (Cl) for perchlorobenzenes (Table 2).

The new atom types (termed protocol P4) were tested for the compounds from the SAMPL3 data set with $\geq 4$ chlorine atoms on an aromatic ring (Table 1 and Figure 4). Gratifyingly, the RMSE for these outlier compounds improved from $4.29 \mathrm{kcal} \cdot \mathrm{mol}^{-1}$ using P1 to $1.22 \mathrm{kcal} \cdot \mathrm{mol}^{-1}$ using P4. Therefore, using the appropriate OPLS-AA parameters (the original force field parameters and the ones proposed in this work), the hydration free energies for all compounds from the SAMPL3 dataset could be calculated with a RMSE of $1.0 \mathrm{kcal} \cdot \mathrm{mol}^{-1}$.

\section{Conclusion}

In this study, hydration free energies for the SAMPL3 dataset were predicted using molecular dynamics simulations with the OPLS-AA force field. Three different protocols for charge generation were used (the original OPLS-AA charges and two variants of QM-derived charges). One of the QM-derived protocols gave the best results, leading to a RMSE value of $1.2 \mathrm{kcal} \cdot \mathrm{mol}^{-1}$ for the whole dataset, whereas the original OPLS-AA charges yielded a RMSE of $2.4 \mathrm{kcal} \cdot \mathrm{mol}^{-1}$. The largest contribu- 
tion to this RMSE was due to compounds with four or more chlorine atoms on the aromatic ring. A new OPLS-AA atom type was developed for these difficult compounds, which allowed a substantial improvement in prediction error. Consequently, using the appropriate OPLS-AA parameters (the original force field parameters and the ones proposed in this work) we are now able to compute the hydration free energies for the whole SAMPL3 dataset with a RMSE of $1.0 \mathrm{kcal} \cdot \mathrm{mol}^{-1}$.

Acknowledgements This work was carried out, in part, under the HPC-EUROPA2 project (project number: 228398) with the support of the European Commission Capacities Area-Research Infrastructures Initiative.

\section{References}

1. Guthrie JP (2009) A blind challenge for computational solvation free energies: introduction and overview. J Phys Chem B 113(14):4501-7, DOI $10.1021 / j p 806724 \mathrm{u}$

2. Geballe MT, Skillman AG, Nicholls A, Guthrie JP, Taylor PJ (2010) The SAMPL2 blind prediction challenge: introduction and overview. J Comput Aided Mol Des 24(4):259-279, DOI 10.1007/s10822-010-9350-8

3. Klimovich PV, Mobley DL (2010) Predicting hydration free energies using allatom molecular dynamics simulations and multiple starting conformations. J Comput Aided Mol Des 24(4):307-316, DOI 10.1007/s10822-010-9343-7

4. Mackerell AD (2004) Empirical force fields for biological macromolecules: Overview and issues. J Comp Chem 25(13):1584-1604, DOI 10.1002/jcc.20082

5. Jorgensen WL, Tirado-Rives J (2005) Potential energy functions for atomic-level simulations of water and organic and biomolecular systems. Proc Natl Acad Sci USA 102(19):6665-6670, DOI 10.1073/pnas.0408037102

6. van Gunsteren WF, Bakowies D, Baron R, Chandrasekhar I, Christen M, Daura X, Gee P, Geerke DP, Glattli A, Hunenberger PH, Kastenholz MA, Oostenbrink C, Schenk M, Trzesniak D, van der Vegt NFA, Yu HB (2006) Biomolecular modeling: Goals, problems, perspectives. Angew Chem Int Ed 45(25):4064-4092, DOI 10.1002/anie.200502655

7. Helms V, Wade R (1997) Free energies of hydration from thermodynamic integration: Comparison of molecular mechanics force fields and evaluation of calculation accuracy. J Comput Chem 18(4):449-462, DOI 10.1002/(SICI)1096987X(199703)18:4<449::AID-JCC1>3.0.CO;2-T

8. Geerke DP, van Gunsteren WF (2006) Force field evaluation for biomolecular simulation: free enthalpies of solvation of polar and apolar compounds in various solvents. ChemPhysChem 7(3):671-8, DOI 10.1002/cphc.200500510

9. Shirts MR, Pande VS (2005) Solvation free energies of amino acid side chain analogs for common molecular mechanics water models. J Chem Phys 122(13): 134,508, DOI 10.1063/1.1877132

10. Mobley DL, Dumont E, Chodera JD, Dill KA (2007) Comparison of charge models for fixed-charge force fields: Small-molecule hydration free energies in explicit solvent. J Phys Chem B 111(9):2242-2254, DOI 10.1021/jp0667442 
11. Mobley DL, Bayly CI, Cooper MD, Dill KA (2009) Predictions of hydration free energies from all-atom molecular dynamics simulations. J Phys Chem B 113(14):4533-4537, DOI 10.1021/jp806838b

12. Mobley DL, Bayly CI, Cooper MD, Shirts MR, Dill KA (2009) Small molecule hydration free energies in explicit solvent: An extensive test of fixedcharge atomistic simulations. J Chem Theory Comput 5(2):350-358, DOI $10.1021 /$ ct800409d

13. Sulea T, Corbeil C, Purisima E (2010) Rapid prediction of solvation free energy. 1. an extensive test of linear interaction energy (lie). J Chem Theory Comput 6(5):1608-1621, DOI 10.1021/ct9006025

14. Purisima EO, Corbeil CR, Sulea T (2010) Rapid prediction of solvation free energy. 3. application to the sampl2 challenge. J Comput Aided Mol Des 24(4):373-83, DOI 10.1007/s10822-010-9341-9

15. Shivakumar D, Williams J, Wu Y, Damm W, Shelley J, Sherman W (2010) Prediction of absolute solvation free energies using molecular dynamics free energy perturbation and the OPLS force field. J Chem Theory Comput 6(5):1509-1519, DOI 10.1021/ct900587b

16. Baker C, Lopes P, Zhu X, Roux B, MacKerell Jr A (2010) Accurate calculation of hydration free energies using pair-specific lennard-jones parameters in the charmm drude polarizable force field. J Chem Theory Comput 6(4):1181-1198, DOI 10.1021/ct9005773

17. Henschler D (1994) Toxicity of chlorinated organic compounds: effects of the introduction of chlorine in organic molecules. Angew Chem Int Ed Engl 33(19):1920-1935, DOI 10.1002/anie.199419201

18. Paulsen M, Straatsma T (1996) Chlorinated ethanes in aqueous solution: parameterization based on thermodynamics of hydration. Chem Phys Lett 259(12):142-145, DOI 10.1016/0009-2614(96)00729-4

19. Ratkova E, Fedorov M (2011) Combination of RISM and cheminformatics for efficient predictions of hydration free energy of polyfragment molecules: Application to a set of organic pollutants. J Chem Theory Comput 7(5):1450-1457, DOI 10.1021/ct100654h

20. Kaminski G, Duffy E, Matsui T, Jorgensen W (1994) Free energies of hydration and pure liquid properties of hydrocarbons from the OPLS all-atom model. J Phys Chem 98(49):13,077-13,082, DOI 10.1021/j100100a043

21. Jorgensen WL, Maxwell DS, Tirado-Rives J (1996) Development and testing of the OPLS all-atom force field on conformational energetics and properties of organic liquids. J Am Chem Soc 118(45):11,225-11,236, DOI 10.1021/ja9621760

22. Damm W, Frontera A, Tirado-Rives J, Jorgensen W (1997) OPLS all-atom force field for carbohydrates. J Comput Chem 18(16):1955-1970, DOI 10.1002/(SICI)1096-987X(199712)18:16<1955::AID-JCC1>3.0.CO;2-L

23. Jorgensen WL, McDonald NA (1998) Development of an all-atom force field for heterocycles. Properties of liquid pyridine and diazenes. J Mol Struct THEOCHEM 424(1-2):145-155, DOI 10.1016/S0166-1280(97)00237-6

24. McDonald NA, Jorgensen WL (1998) Development of an all-atom force field for heterocycles. Properties of liquid pyrrole, furan, diazoles, and oxazoles. J Phys Chem B 102(41):8049-8059, DOI 10.1021/jp981200o 
25. Rizzo RC, Jorgensen WL (1999) OPLS all-atom model for amines: Resolution of the amine hydration problem. J Am Chem Soc 121(20):4827-4836, DOI 10.1021/ja984106u

26. Kaminski GA, Friesner RA, Tirado-Rives J, Jorgensen WL (2001) Evaluation and reparametrization of the OPLS-AA force field for proteins via comparison with accurate quantum chemical calculations on peptides. J Phys Chem B 105(28):6474-6487, DOI 10.1021/jp003919d

27. Watkins EK, Jorgensen WL (2001) Perfluoroalkanes: Conformational analysis and liquid-state properties from ab initio and Monte Carlo calculations. J Phys Chem A 105(16):4118-4125, DOI 10.1021/jp004071w

28. Price M, Ostrovsky D, Jorgensen W (2001) Gas-phase and liquid-state properties of esters, nitriles, and nitro compounds with the OPLS-AA force field. J Comput Chem 22(13):1340-1352, DOI 10.1002/jcc.1092

29. Kony D, Damm W, Stoll S, Van Gunsteren W (2002) An improved OPLSAA force field for carbohydrates. J Comput Chem 23(15):1416-1429, DOI $10.1002 /$ jcc. 10139

30. Kahn K, Bruice T (2002) Parameterization of OPLS-AA force field for the conformational analysis of macrocyclic polyketides. J Comput Chem 23(10):977996, DOI 10.1002/jcc.10051

31. Thomas L, Christakis T, Jorgensen W (2006) Conformation of alkanes in the gas phase and pure liquids. J Phys Chem B 110(42):21,198-21,204, DOI 10.1021/jp064811m

32. Jorgensen W, Jensen K, Alexandrova A (2007) Polarization effects for hydrogenbonded complexes of substituted phenols with water and chloride ion. J Chem Theory Comput 3(6):1987-1992, DOI 10.1021/ct7001754

33. Xu Z, Luo HH, Tieleman DP (2007) Modifying the opls-aa force field to improve hydration free energies for several amino acid side chains using new atomic charges and an off-plane charge model for aromatic residues. J Comput Chem 28(3):689-97, DOI 10.1002/jcc.20560

34. Hess B, Kutzner C, van der Spoel D, Lindahl E (2008) Gromacs 4: Algorithms for highly efficient, load-balanced, and scalable molecular simulation. J Chem Theory Comput 4(3):435-447, DOI 10.1021/ct700301q

35. Michaud-Agrawal N, Denning EJ, Woolf TB, Beckstein O (2011) MDAnalysis: A toolkit for the analysis of molecular dynamics simulations. J Comput Chem 32:2319-2327, DOI 10.1002/jcc.21787

36. Frisch MJ, Trucks GW, Schlegel HB, Scuseria GE, Robb MA, Cheeseman JR, Montgomery JA Jr, Vreven T, Kudin KN, Burant JC, Millam JM, Iyengar SS, Tomasi J, Barone V, Mennucci B, Cossi M, Scalmani G, Rega N, Petersson GA, Nakatsuji H, Hada M, Ehara M, Toyota K, Fukuda R, Hasegawa J, Ishida M, Nakajima T, Honda Y, Kitao O, Nakai H, Klene M, Li X, Knox JE, Hratchian HP, Cross JB, Bakken V, Adamo C, Jaramillo J, Gomperts R, Stratmann RE, Yazyev O, Austin AJ, Cammi R, Pomelli C, Ochterski JW, Ayala PY, Morokuma K, Voth GA, Salvador P, Dannenberg JJ, Zakrzewski VG, Dapprich S, Daniels AD, Strain MC, Farkas O, Malick DK, Rabuck AD, Raghavachari K, Foresman JB, Ortiz JV, Cui Q, Baboul AG, Clifford S, Cioslowski J, Stefanov BB, Liu G, Liashenko A, Piskorz P, Komaromi I, Martin RL, Fox DJ, Keith T, Al-Laham 
MA, Peng CY, Nanayakkara A, Challacombe M, Gill PMW, Johnson B, Chen W, Wong MW, Gonzalez C, Pople JA (2004) Gaussian 03, Revision E.01. Gaussian, Inc., Wallingford, CT

37. Jorgensen W, Ulmschneider J, Tirado-Rives J (2004) Free energies of hydration from a generalized born model and an all-atom force field. J Phys Chem B 108(41):16,264-16,270, DOI 10.1021/jp0484579

38. Jorgensen WL, Chandrasekhar J, Madura JD, Impey RW, Klein ML (1983) Comparison of simple potential functions for simulating liquid water. J Chem Phys 79(2):926-935, DOI 10.1063/1.445869

39. Essman U, Perela L, Berkowitz ML, Darden T, Lee H, Pedersen LG (1995) A smooth particle mesh Ewald method. J Chem Phys 103:8577-8592, DOI 10.1063/1.470117

40. Hess B (2008) P-LINCS: A parallel linear constraint solver for molecular simulation. J Chem Theory Comput 4(1):116-122, DOI 10.1021/ct700200b

41. Jorge M, Garrido N, Queimada A, Economou I, Macedo E (2010) Effect of the integration method on the accuracy and computational efficiency of free energy calculations using thermodynamic integration. J Chem Theory Comput 6(4):1018-1027, DOI 10.1021/ct900661c

42. Frenkel D, Smit B (2002) Understanding Molecular Simulations, 2nd edn. Academic Press, San Diego 

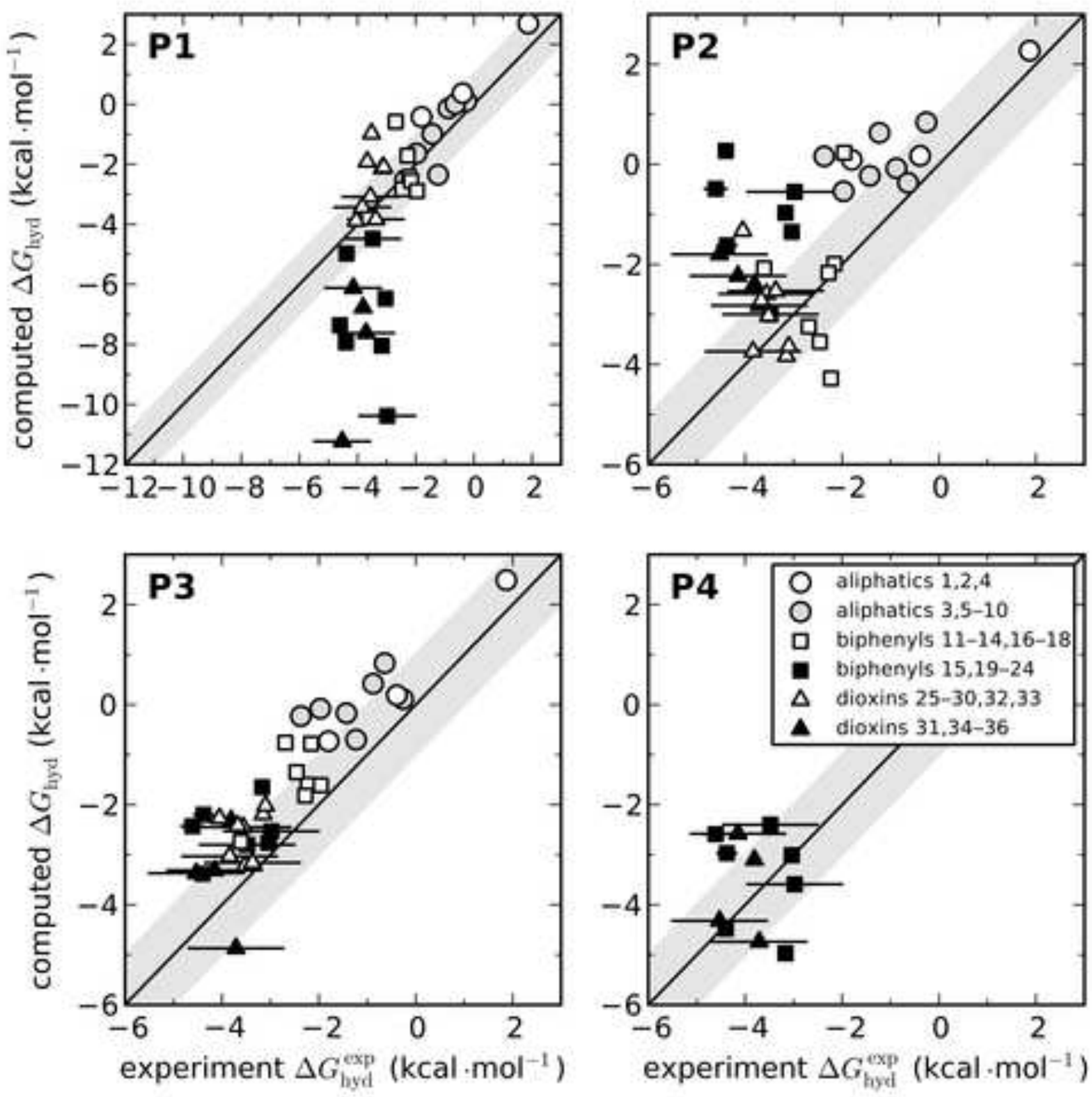
A

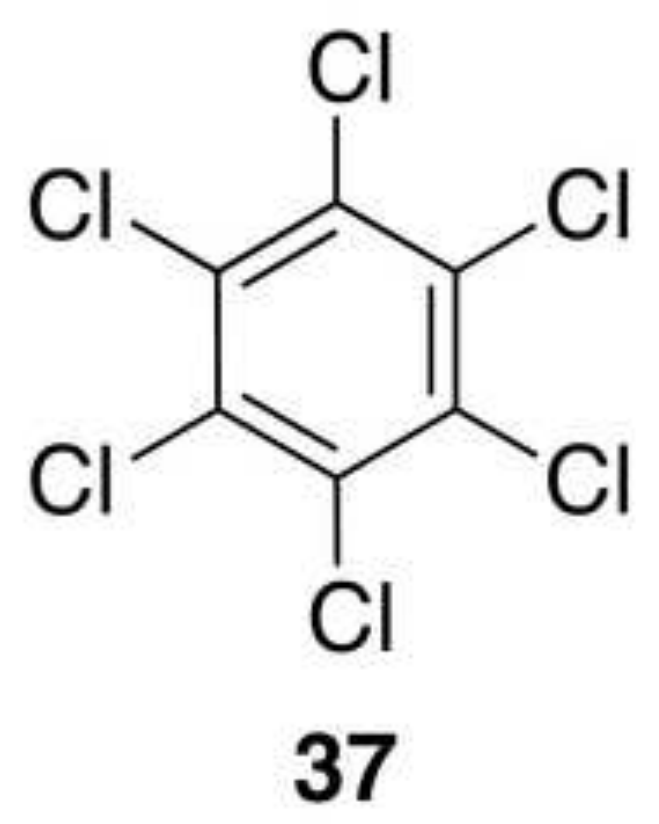

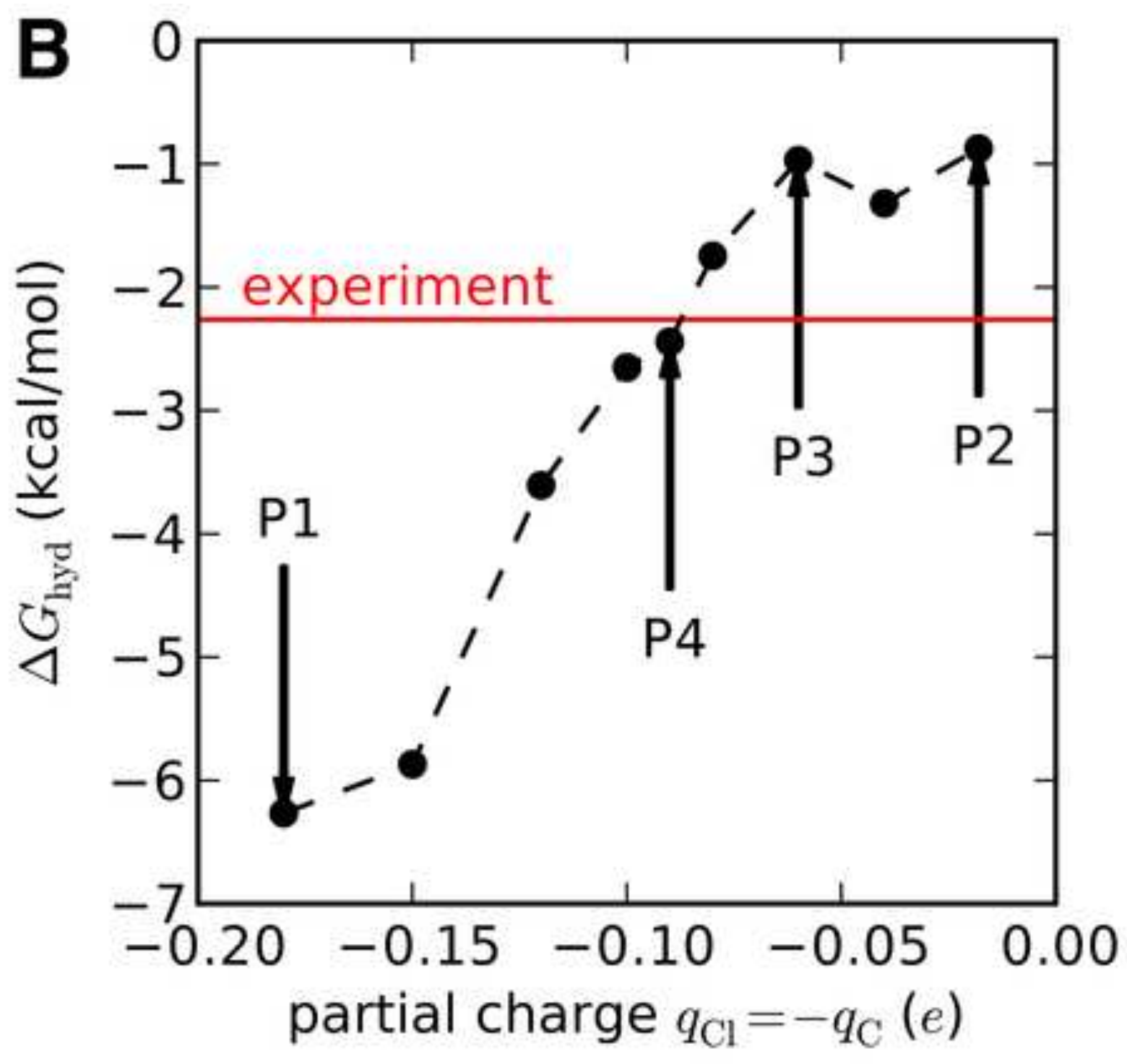




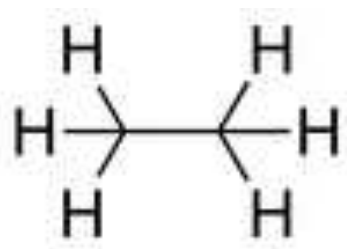

1

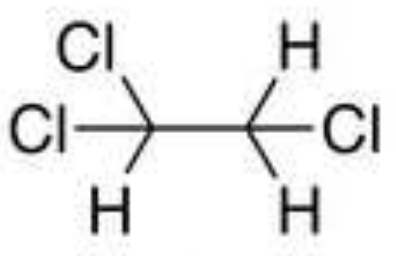

6

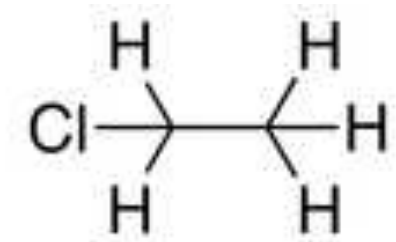

2

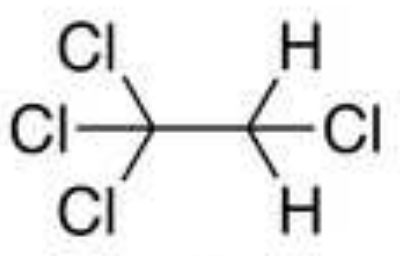

7

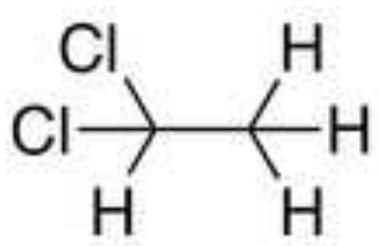

3

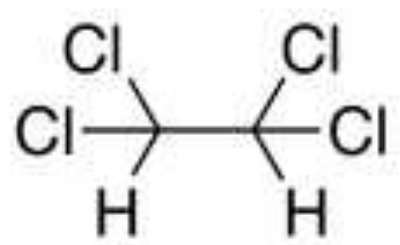

8

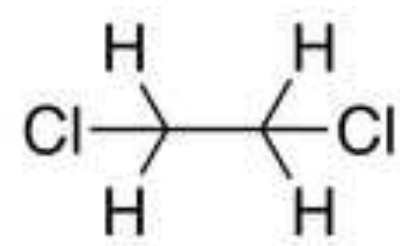

4

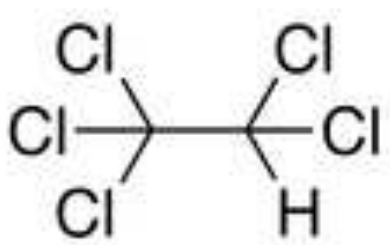

9
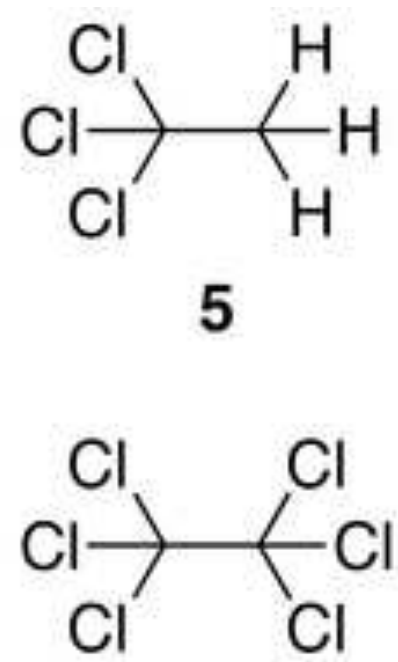

10 


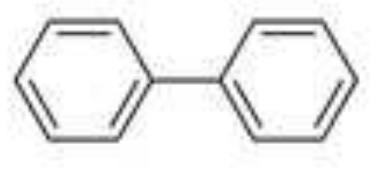

11

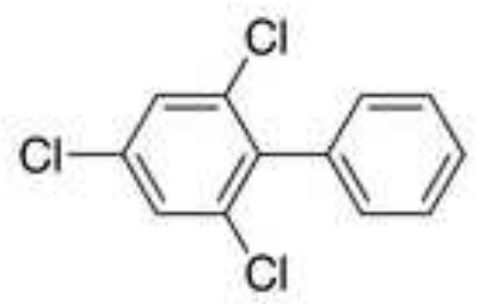

14

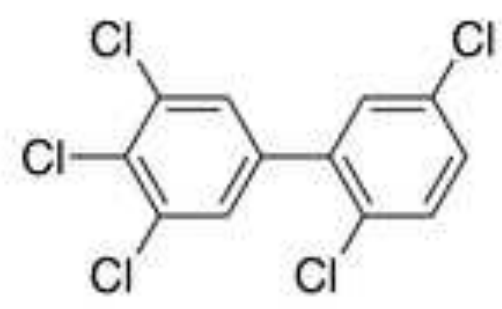

17

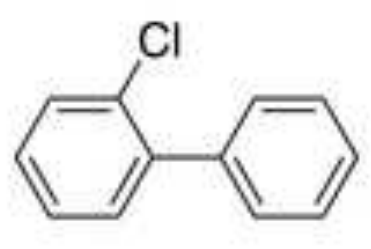

12
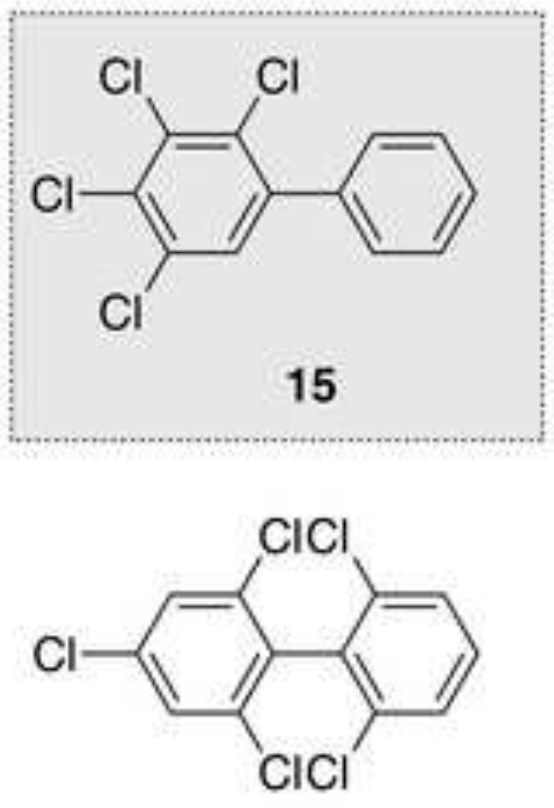

18

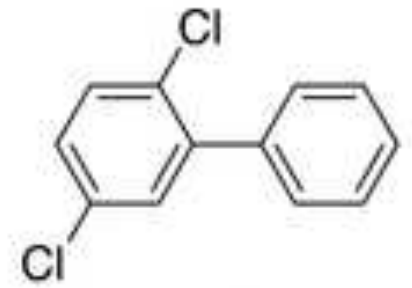

13

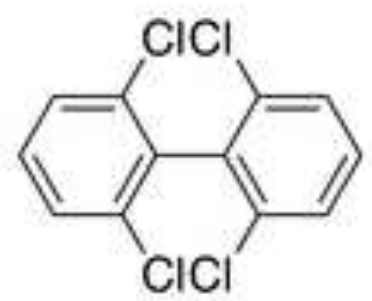

16

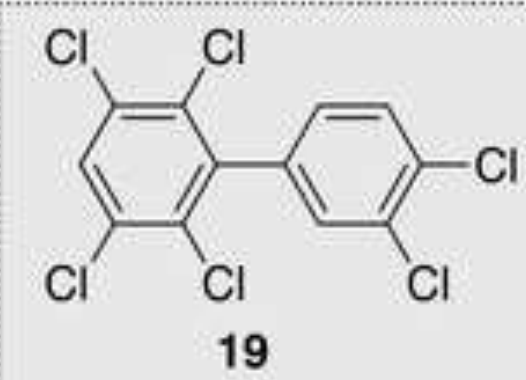

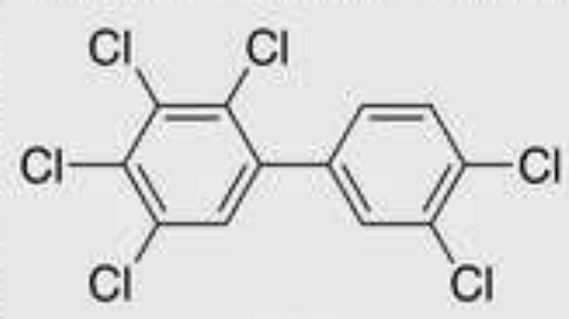

20

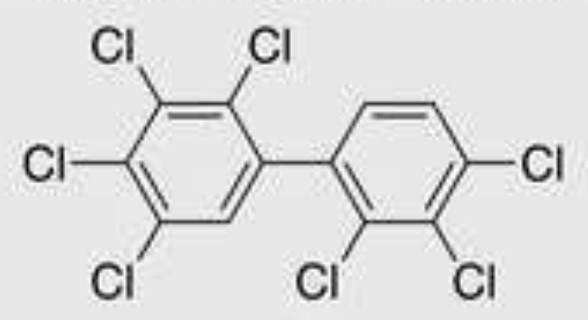

21

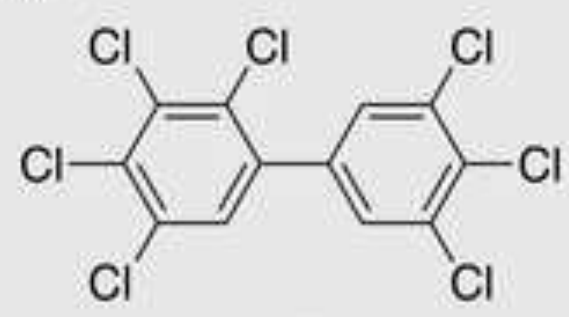

22

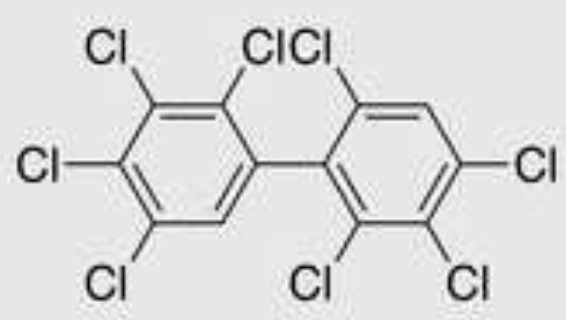

23

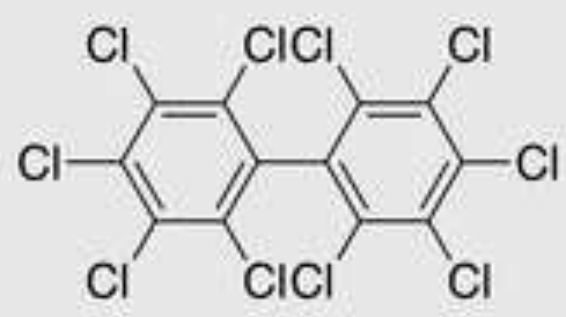

24 
<smiles>c1ccc2c(c1)Oc1ccccc1O2</smiles>

25<smiles>Clc1cc2c(cc1Cl)Oc1ccccc1O2</smiles>

28

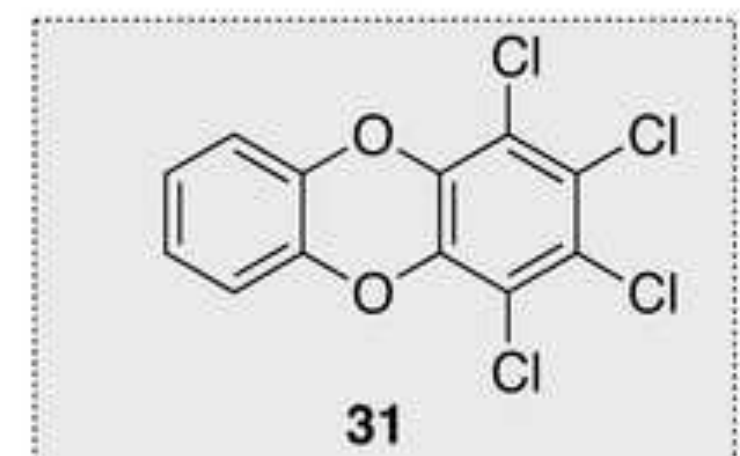

29<smiles>Clc1ccc2c(c1)Oc1cc(Cl)c(Cl)c(Cl)c1O2</smiles><smiles>Clc1ccc2c(c1)Oc1ccccc1O2</smiles>

27<smiles>Clc1cc(Cl)c2c(c1Cl)Oc1ccccc1O2</smiles>

30<smiles>Clc1ccc2c(c1)Oc1c(Cl)c(Cl)c(Cl)c(Cl)c1O2</smiles>

34<smiles>Clc1c(Cl)c(Cl)c2c(c1Cl)Oc1c(Cl)c(Cl)c(Cl)c(Cl)c1O2</smiles> 


\section{Supplementary Information}

Prediction of hydration free energies for aliphatic and aromatic chloro derivatives using molecular dynamics simulations with the OPLS-AA force field

\section{Oliver Beckstein · Bogdan I. Iorga}

\section{NVT correction}

In order to compute a Gibbs hydration free energy $\Delta G_{\text {hyd }}$ (at constant $P$ ) from a Helmholtz hydration free energy $\Delta A_{\text {hyd }}$ (at constant $V$ ) a correction

$$
\Delta W:=\Delta G_{\mathrm{hyd}}-\Delta A_{\mathrm{hyd}}
$$

can be added to $\Delta A_{\text {hyd }}$. In the following the correction $\Delta W$ is derived from thermodynamic arguments. $\Delta W$ equals the difference in work required to create a cavity in the solvent of the size of the solute under conditions of either constant pressure $P$ or constant volume $V$,

$$
\Delta W=W_{N P T}-W_{N V T},
$$

while both particle number $N$ and temperature $T$ are held constant.

For the process at constant pressure creating a volume of size $v_{S}$ simply requires the work against the external pressure and the formation of a liquid-vacuum interface of area $A$,

$$
W_{N P T}=\int_{0}^{v_{s}} d V P+\gamma A=v_{s} P+\gamma A .
$$

(The surface term with the surface tension $\gamma$ is of no importance in the following discussion as it will cancel exactly in the final result.)

\footnotetext{
O. Beckstein of Oxford, Oxford OX1 3QU, UK 1504, USA

B.I. Iorga 1 Avenue de la Terrasse, 91198 Gif-sur-Yvette, France

Tel.: +33169823094

Fax: +3316907 7247

E-mail: bogdan.iorga@icsn.cnrs-gif.fr
}

Structural Bioinformatics and Computational Biochemistry Unit, Department of Biochemistry, University

Present address: Department of Physics, Arizona State University, P.O. Box 871504, Tempe, AZ $85287-$

Institut de Chimie des Substances Naturelles, CNRS UPR 2301, Centre de Recherche de Gif-sur-Yvette, 
Growing a solute into neat solvent If pure solvent initially fills a constant volume $V_{0}$, work must be expended to compress the solvent when a cavity of volume $v_{s}$ is introduced. The work $W_{N V T}$ to change the solvent volume from $V_{0}$ to $V_{0}-v_{s}$ at constant temperature $T$ is

$$
W_{N V T}=-\int_{V_{0}}^{V_{0}-v_{s}} d V P(T, V, N)+\gamma A=\int_{0}^{v_{s}} d v P\left(T, V_{0}-v, N\right)+\gamma A .
$$

$P(T, V, N)$ is the equation of state of the solvent. Here we are interested in a process at constant particle number $N$ and in order to simplify the notation, references to $N$ are dropped in the following. Assuming that the change in solvent volume is small (small solute, large simulation box volume $V_{0}$, i.e. $v_{s} / V_{0} \ll 1$ ) we can expand the equation of state around $V_{0}$ as

$$
P\left(T, V_{0}-v\right)=P\left(T, V_{0}\right)+\sum_{n=1}^{\infty} \frac{(-v)^{n}}{n !} \partial_{V}^{n} P\left(V_{0}, T\right),
$$

where $\partial_{V}^{n} P\left(V_{0}, T\right)$ denotes the $n$-th partial derivative with respect to volume at constant temperature $T,\left(\partial^{n} P / \partial V^{n}\right)_{T}$, evaluated at $V=V_{0}$. These partial derivatives can be expressed in terms of the compressibility at constant temperature

$$
\kappa_{T}:=-\frac{1}{V}\left(\frac{\partial V}{\partial P}\right)_{T}
$$

by using the identity for partial derivatives $\partial_{V} P(T, V, N)=\left[\partial_{P} V(T, P, N)\right]^{-1}$. In particular, the first derivative is

$$
\partial_{V} P(T, V, N)=-\kappa_{T}^{-1} V^{-1}
$$

Under the assumption that the compressibility does not depend on $V$, ${ }^{1}$ i.e.

$$
\partial_{V} \kappa_{T}(T, V, N)=0,
$$

all derivatives can be evaluated as

$$
\partial_{V}^{n} P(T, V)=\kappa_{T}^{-1}(-1)^{n}(n-1) ! V^{-n}
$$

and the Taylor expansion Eq. ?? becomes

$$
\begin{aligned}
P\left(T, V_{0}-v\right) & =P\left(T, V_{0}\right)+\kappa_{T}^{-1} \sum_{n=1}^{\infty} \frac{(-v)^{n}}{n !}(-1)^{n}(n-1) ! V_{0}^{-n} \\
& =P\left(T, V_{0}\right)+\kappa_{T}^{-1} \sum_{n=1}^{\infty} \frac{1}{n}\left(\frac{v}{V_{0}}\right)^{n} \\
& =P\left(T, V_{0}\right)-\kappa_{T}^{-1} \ln \left(1-\frac{v}{V_{0}}\right)
\end{aligned}
$$

1 The assumption of volume independence is equivalent to the assumption that for fixed $N, \kappa_{T}$ does not depend on the density $N / V$. 

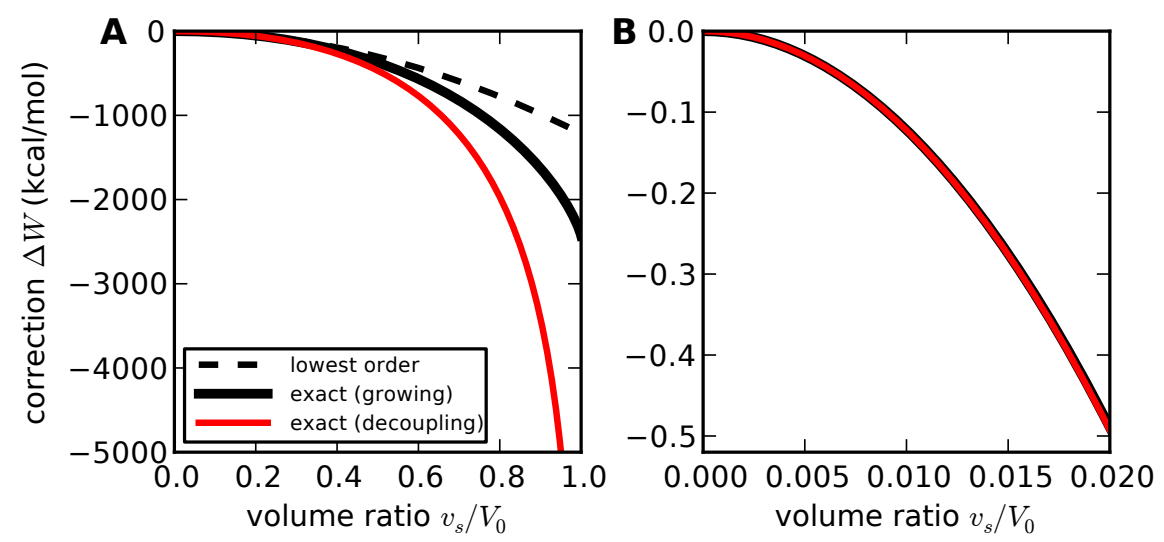

Fig. S1 Exact correction $\Delta W$ Eq. ?? for growing a solute into water (black straight line, "exact (growing)"), for decoupling a solute, Eq. ?? (red straight line, "exact (decoupling)"), and lowest order correction Eq. ?? (dashed, "lowest order"), plotted for $V_{0}=10 \mathrm{~nm}^{3}$ and $\kappa_{T}=5.9 \times 10^{-5} \mathrm{bar}^{-1}$. A: over the full range of volume ratios $v_{s} / V_{0}$. B: range of volume ratios up to $2 \%$ as occuring in this work where the two exact corrections are virtually indistinguishable from the lowest order correction.

where the series expansion for $\ln (1-x)$ was used in the last step and the volume ratio is restricted to $0 \leq v_{s} / V_{0}<1$. Integrating Eq. ?? with Eq. ?? yields

$$
W_{N V T}=v_{s} P\left(T, V_{0}\right)-\kappa_{T}^{-1} V_{0}\left[\left(\frac{v_{s}}{V_{0}}-1\right) \ln \left(1-\frac{v_{s}}{V_{0}}\right)-\frac{v_{s}}{V_{0}}\right], \quad \text { with } 0 \leq \frac{v_{s}}{V_{0}}<1 .
$$

If the volume $V_{0}$ of the $N V T$ system is chosen so that its pressure $P\left(T, V_{0}\right)$ equals the pressure $P$ of the NPT system then the correction Eq. ?? is, using Equations ?? and $? ?,{ }^{2}$

$$
\Delta W=-\kappa_{T}^{-1} V_{0}\left[\frac{v_{s}}{V_{0}}+\left(1-\frac{v_{s}}{V_{0}}\right) \ln \left(1-\frac{v_{s}}{V_{0}}\right)\right] .
$$

The correction is only defined for $0 \leq v_{s} / V_{0}<1$ and in this range $\Delta W \leq 0$. Without a solute $\left(v_{s}=0\right)$ the correction is zero but for the pathological case $\lim _{v_{s} / V_{0} \rightarrow 1} \Delta W=$ $-\kappa_{T}^{-1} V_{0}$ it is still finite (Fig. ??). In this regime, the solute would take up all the space available and the solvent would need to be compressed into an infinitely small volume. A finite value of $\Delta W$ indicates a clear breakdown of the assumption (Eq. ??) that the compressibility $\kappa_{T}$ is independent of the volume (or the density) of the solvent phase. The actual range of applicability of Eq. ?? is not immediately obvious although for the volume ratios $v_{s} / V_{0}<0.02$ encountered in this work we always observed an improvement in computed and corrected values with regard to experiment.

Decoupling of a solvated solute In the previous section the correction $\Delta W$ was derived for the case when a solute is inserted into a fixed volume $V_{0}$ with water at

\footnotetext{
2 If the volume is not matched to the pressure then an additional term $v_{S}\left[P-P\left(T, V_{0}, N\right)\right]$ is included in Eq. ??. This term is comparatively small as $1 \mathrm{bar}=0.060221415 \mathrm{~kJ} \cdot \mathrm{mol}^{-1} \cdot \mathrm{nm}^{-3}$.
} 
conditions matching the equivalent system at constant pressure and solvent density. Using a similar approach one can also derive the correction for the case when a solute is decoupled (removed) from a system with volume $V_{0}$ that corresponds to the system with the same density $N /\left(V_{0}-v_{s}\right)$ and constant pressure $P$. The final result is

$$
\Delta W=\kappa_{T}^{-1} V_{0}\left[\frac{v_{s}}{V_{0}}+\ln \left(1-\frac{v_{s}}{V_{0}}\right)\right] .
$$

It reduces to the same lowest order approximation as Eq. ?? (see Eq. ?? below) and is $\leq 0$ in its range of validity (Fig. ??). Unlike Eq. ??, it tends to $-\infty$ for $v_{s} \rightarrow V_{0}$.

In order to correct the $N V T$ simulation results $\Delta A_{\text {hyd }}$ in this work, we applied the correction Eq. ?? as $\Delta G_{\text {hyd }}=\Delta A_{\text {hyd }}+\Delta W$ with the compressibility $\kappa_{T}=5.9 \times$ $10^{-5}$ bar $^{-1}$ for TIP4P water at standard conditions $\left(1.013\right.$ bar and $\left.25^{\circ} \mathrm{C}\right)$ [? ] (although a somewhat different value of $4.93 \pm 0.06 \times 10^{-5} \mathrm{bar}^{-1}$ has been reported at $\left.10^{\circ} \mathrm{C}[?]\right)$. Data for all studied compounds is listed in Table ??.

The experimentally determined compressibility of water is very small $\left(\kappa_{T}=\right.$ $4.5248 \times 10^{-5} \mathrm{bar}^{-1}=7.5136 \times 10^{-4} \mathrm{~kJ}^{-1} \cdot \mathrm{mol} \cdot \mathrm{nm}^{3}$ at $25^{\circ} \mathrm{C}$ temperature and $1 \mathrm{~atm}$ pressure [?]). It only varies weakly with the specific volume (or its reciprocal, the specific density) in a range where the specific volume changes by less than $4 \%$ from $1.002961 \mathrm{~cm}^{3} \cdot \mathrm{g}^{-1}$ (at $1.013 \mathrm{bar}$ ) to $0.963507 \mathrm{~cm}^{3} \cdot \mathrm{g}^{-1}$ (at 1014 bar where $\kappa_{T}=3.5675 \times 10^{-5}$ bar $^{-1}$ ) [? ]. This observation provides the motivation for the assumption Eq. ?? that the compressibility is independent from the density.

Lowest order approximation The lowest order approximation of Eq. ?? or Eq. ?? is instructive as it shows the essential structure of the correction. Ignoring the higher order terms in Eq. ?? leads to

$$
P\left(T, V_{0}-v\right)=P\left(T, V_{0}\right)-v \frac{1}{-\kappa_{T} V_{0}}+\cdots
$$

and hence

$$
W_{N V T}=v_{s} P\left(T, V_{0}\right)+\frac{1}{2} v_{s}^{2} \kappa_{T}^{-1} V_{0}^{-1} .
$$

The correction Eq. ?? is

$$
\Delta W=-\frac{1}{2} v_{s}^{2} \kappa_{T}^{-1} V_{0}^{-1}=-\frac{1}{2} \kappa_{T}^{-1} V_{0}\left(\frac{v_{s}}{V_{0}}\right)^{2}
$$

to lowest non-vanishing order in the solute volume. $\Delta W$ depends on the solute volume $v_{s}$ at least quadratically. Larger simulation boxes $V_{0}$ decrease the magnitude of the correction because less compression of the liquid is required to make space for the solute.

With the computed value of $\kappa_{T}=5.9 \times 10^{-5} \mathrm{bar}^{-1}$ for TIP4P water at standard conditions [?] (compared to the experimental value $4.5248 \times 10^{-5} \mathrm{bar}^{-1}$ [? ]), the lowest order correction is calculated as

$$
\Delta W=-508.5 \mathrm{~kJ} \cdot \mathrm{mol}^{-1} \cdot \mathrm{nm}^{-3} v_{s}^{2} V_{0}^{-1} .
$$

Eq. ?? is a good approximation to both Eq. ?? and Eq. ?? for small volume ratios, as the difference is on the order of $\mathscr{O}\left(\left(\frac{v_{s}}{V_{0}}\right)^{3}\right)$ (Fig. ??). 
Table S1 NVT correction for compounds studied. The volume $v_{s}$ of each solute was computed with UCSF Chimera [?] and the volume of the dodecahedral simulation cell $V_{0}$ was calculated with the Gromacs tool editconf. The correction $\Delta W$ was calculated according to Eq. ??.

\begin{tabular}{rrrrr}
\hline id & $v_{s}\left(\mathrm{~nm}^{3}\right)$ & $V_{0}\left(\mathrm{~nm}^{3}\right)$ & $v_{s} / V_{0}$ & $\Delta W\left(\mathrm{kcal} \cdot \mathrm{mol}^{-1}\right)$ \\
\hline $\mathbf{1}$ & 0.033 & 8.23 & $0.40 \%$ & -0.016 \\
$\mathbf{2}$ & 0.058 & 9.08 & $0.64 \%$ & -0.045 \\
$\mathbf{3}$ & 0.082 & 9.00 & $0.91 \%$ & -0.092 \\
$\mathbf{4}$ & 0.083 & 9.83 & $0.84 \%$ & -0.085 \\
$\mathbf{5}$ & 0.106 & 9.07 & $1.16 \%$ & -0.151 \\
$\mathbf{6}$ & 0.106 & 8.93 & $1.19 \%$ & -0.155 \\
$\mathbf{7}$ & 0.129 & 9.84 & $1.31 \%$ & -0.207 \\
$\mathbf{8}$ & 0.130 & 10.01 & $1.30 \%$ & -0.207 \\
$\mathbf{9}$ & 0.153 & 10.10 & $1.51 \%$ & -0.283 \\
$\mathbf{1 0}$ & 0.175 & 9.84 & $1.78 \%$ & -0.381 \\
$\mathbf{1 1}$ & 0.138 & 17.40 & $0.79 \%$ & -0.134 \\
$\mathbf{1 2}$ & 0.161 & 17.00 & $0.95 \%$ & -0.187 \\
$\mathbf{1 3}$ & 0.184 & 17.51 & $1.05 \%$ & -0.238 \\
$\mathbf{1 4}$ & 0.210 & 18.48 & $1.14 \%$ & -0.293 \\
$\mathbf{1 5}$ & 0.230 & 18.45 & $1.25 \%$ & -0.351 \\
$\mathbf{1 6}$ & 0.241 & 17.22 & $1.40 \%$ & -0.413 \\
$\mathbf{1 7}$ & 0.256 & 18.41 & $1.39 \%$ & -0.435 \\
$\mathbf{1 8}$ & 0.265 & 18.67 & $1.42 \%$ & -0.460 \\
$\mathbf{1 9}$ & 0.281 & 18.34 & $1.53 \%$ & -0.529 \\
$\mathbf{2 0}$ & 0.277 & 19.88 & $1.39 \%$ & -0.474 \\
$\mathbf{2 1}$ & 0.302 & 19.76 & $1.53 \%$ & -0.568 \\
$\mathbf{2 2}$ & 0.301 & 19.94 & $1.51 \%$ & -0.559 \\
$\mathbf{2 3}$ & 0.331 & 19.78 & $1.67 \%$ & -0.681 \\
$\mathbf{2 4}$ & 0.376 & 19.84 & $1.90 \%$ & -0.878 \\
$\mathbf{2 5}$ & 0.145 & 17.93 & $0.81 \%$ & -0.143 \\
$\mathbf{2 6}$ & 0.169 & 17.47 & $0.97 \%$ & -0.200 \\
$\mathbf{2 7}$ & 0.168 & 18.85 & $0.89 \%$ & -0.183 \\
$\mathbf{2 8}$ & 0.191 & 18.80 & $1.01 \%$ & -0.237 \\
$\mathbf{2 9}$ & 0.192 & 20.22 & $0.95 \%$ & -0.222 \\
$\mathbf{3 0}$ & 0.217 & 18.71 & $1.16 \%$ & -0.308 \\
$\mathbf{3 1}$ & 0.239 & 18.90 & $1.27 \%$ & -0.371 \\
$\mathbf{3 2}$ & 0.239 & 19.99 & $1.19 \%$ & -0.349 \\
$\mathbf{3 3}$ & 0.249 & 20.14 & $1.24 \%$ & -0.379 \\
$\mathbf{3 4}$ & 0.263 & 20.15 & $1.30 \%$ & -0.420 \\
$\mathbf{3 5}$ & 0.286 & 20.12 & $1.42 \%$ & -0.498 \\
$\mathbf{3 6}$ & 0.332 & 20.00 & $1.66 \%$ & -0.676 \\
$\mathbf{3 7}$ & 0.208 & 12.09 & $1.72 \%$ & -0.439 \\
\hline & & & &
\end{tabular}

\section{Three-dimensional representation of compounds 1-36}

The parameterizations $\mathrm{P} 1-\mathrm{P} 4$ only differ in the partial charges. To provide an overview over the different distribution of those charges, Table 1 shows each compound with the partial charges indicated by colors. Colors range from red (negative values) to blue (positive) with zero charges in white. 
Table S2: Three-dimensional representation of compounds 1-36 (sticks), colored according to the partial charge on each atom.

id

P1

P2

P3

P4

1

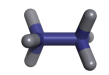

$\&$

$\&$

2
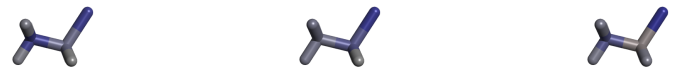

3<smiles>C=C(C)C(C)=CC</smiles><smiles>C=C(C)C(C)=C(C)C</smiles><smiles>C=C(C)C(=C)C</smiles>

4
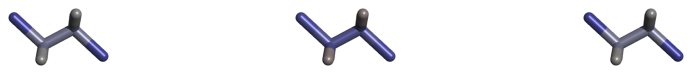

5<smiles>C=C(C)C(=C)C</smiles><smiles>C=C(C)C(=C)C</smiles><smiles>C=C(C)C(=C)C</smiles>

6
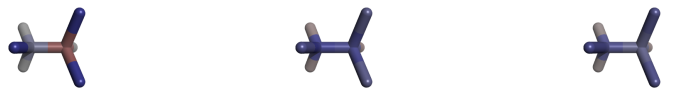

7
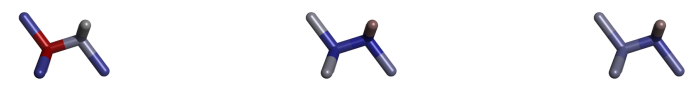

8
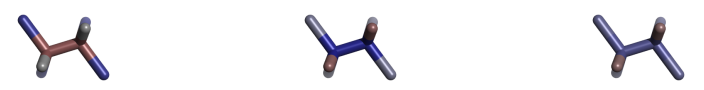
SAMPL3: OPLS-AA hydration free energies from MD

7

id

PI

9

Y

10

H

11

12

13

14

15

16

17
Table S2 - continued

P2

X

H

$x$
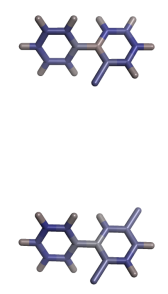

故好
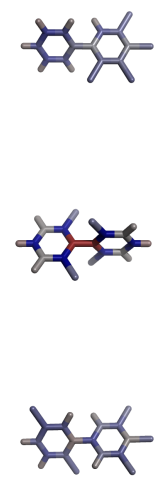

PB

H

H

$2 x$

Pu
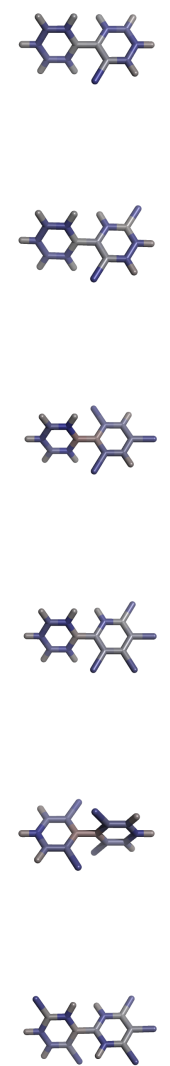
8

Oliver Beckstein, Bogdan I. Iorga

id

P1

18

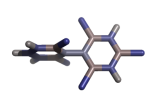

19

20 站格

21 准

22 证证

23

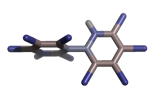

24

25

26
Table S2 - continued

P2

P3

P4
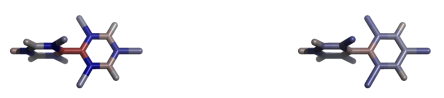

证姑

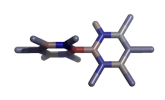

证和

200

sose
证

增

证的

沶

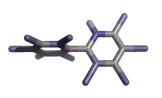

保

坛的

证名

始

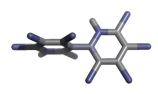

识婂

说话 
id

P1

27

28

sose

29

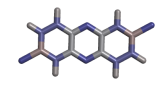

sose

soose

30

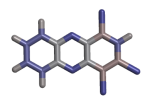<smiles>CC(C)(C)C(C)(C)C(C)(C)C(C)(C)C(C)(C)C</smiles>

rof

31

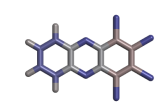

sos<smiles>[Mg]</smiles>

xos<smiles>[Mg]</smiles><smiles>[Mg]</smiles><smiles>[Mg]</smiles>

33<smiles>[Mg]</smiles><smiles>[Mg]</smiles><smiles>[Mg]</smiles>

$34 \quad$<smiles>[Mg]</smiles><smiles>[Mg]</smiles><smiles>[Mg]</smiles>

35<smiles>[Mg]</smiles><smiles>[Mg]</smiles><smiles>[Mg]</smiles><smiles>[Mg]</smiles> 
id

P1

36
Table S2 - continued

P2

P3

P4

top sos 